\title{
MIMO Antenna Selection With Lattice-Reduction-Aided Linear Receivers
}

\author{
Inaki Berenguer and Xiaodong Wang, Member, IEEE
}

\begin{abstract}
A method to improve the performance of multiple-input-multiple-output systems is to employ a large number of antennas and select the optimal subset depending on the specific channel realization. A simple antenna-selection criterion is to choose the antenna subset that maximizes the mutual information. However, when the receiver has finite complexity decoders, this criterion does not necessarily minimize the error rate (ER). Therefore, different selection criteria should be tailored to the specific receiver implementation. In this paper, we develop new antenna-selection criteria to minimize the ER in spatial multiplexing systems with lattice-reduction-aided receivers. We also adapt other known selection criteria, such as maximum mutual information, to this specific receiver. Moreover, we consider adaptive antenna-selection algorithms when the channel is not perfectly known at the receiver but can only be estimated. We present simulation examples to show the ER of the different selection criteria and the convergence of the adaptive algorithms. We also discuss the difference in complexity and performance among them.
\end{abstract}

Index Terms-Adaptive algorithm, antenna selection, lattice reduction, minimum error rate (ER), multiple-input-multiple-output (MIMO).

\section{INTRODUCTION}

M ULTIPLE-INPUT-MULTIPLE-OUTPUT

(MIMO) communication systems have received significant attention during recent years due to the high capacity improvement that they can offer over single-input-single-output (SISO) systems [1], [2]. One concern when using MIMO systems is the amount of hardware required for the radio-frequency (RF) chains attached to each antenna, which consist of expensive hardware blocks such as analog-to-digital converters, mixers, and low-noise power amplifiers. On the other hand, antennas (patch or dipole) are generally cheaper elements. Therefore, an approach to obtain better performance in a MIMO system is to use a fixed number of RF chains and a larger number of antennas than actually being used. Then, given a specific channel realization, a selection algorithm can be implemented to combine the RF chains with the subset of antennas, which gives better performance, e.g., in terms of capacity or error rate (ER).

Manuscript received July 31, 2003; revised December 22, 2003, March 27, 2004, and April 26, 2004. This work was supported in part by the U.S. National Science Foundation (NSF) under Grant CCR-0207550 and by the U.S. Office of Naval Research (ONR) under Grant N00014-03-1-0039. The work of I. Berenguer was supported by a Fulbright Fellowship.

The authors are with the Department of Electrical Engineering, Columbia University, New York, NY 10027 USA (e-mail: ib@ee.columbia.edu; wangx@ee.columbia.edu).

Digital Object Identifier 10.1109/TVT.2004.832389
Various antenna-selection schemes in MIMO systems have been studied in the recent literature. A selection mechanism is proposed in [3], according to which the best subset of transmit or receive antennas is selected based on the mutual information criterion. A suboptimal algorithm that does not need to perform an exhaustive search over all possible subsets is proposed in [4]. A statistical analysis to relate the capacity when the whole set of antennas is used and the channel capacity with subset selection is reported in [5].

Antenna-selection algorithms to minimize the symbol error rate (SER) when orthogonal space-time block codes are used in MIMO systems, are proposed in [6]. Selection algorithms that only assume knowledge of the second-order statistics of the MIMO channel are also presented in [7]. In [8], a study of the impact of antenna selection on the pairwise error probability is conducted. In particular, this work looks at the impact of antenna selection at the receiver on the diversity order and coding gain of the underlying orthogonal space-time code, assuming that the antenna subset maximizing the signal-to-noise ratio (SNR) is selected. This work shows that the diversity gain provided by the code is maintained, whereas the coding gain is reduced. In [9], the authors design space-time codes for two transmit antennas based on the pairwise error probability that perform better when antenna selection at the receiver is used. Antennaselection algorithms at the transmitter are studied in [10] when the Alamouti code and power allocation are implemented using the feedback channel-state information and considering errors in the information feedback to the transmitter.

Antenna selection in spatial multiplexing systems has also been addressed recently. Antenna-selection algorithms that attempt to minimize the bit-error rate (BER) in linear receivers are presented in [11]. In [12], a statistical analysis on the MIMO channel capacity is performed considering spatial multiplexing systems with receive antenna selection. This work proves that the diversity order, defined as the slope between the capacity and outage rate in the region of small outage rates, which is achievable through antenna selection, is the same as that of the full system, which motivates the use of receive antenna selection in spatial multiplexing systems. Other work on MIMO antenna selection is also reported in [13]-[17].

In this paper, we propose receive-antenna-selection algorithms for an arbitrary number of $M_{T}$ transmit antennas and $M_{R}$ receive antennas $\left(M_{R} \geq M_{T}\right)$ when spatial multiplexing systems are used at the transmitter and the recently proposed lattice-reduction-aided (LRA) decoders are used at the receiver. LRA receivers have a complexity similar to the linear receivers, 


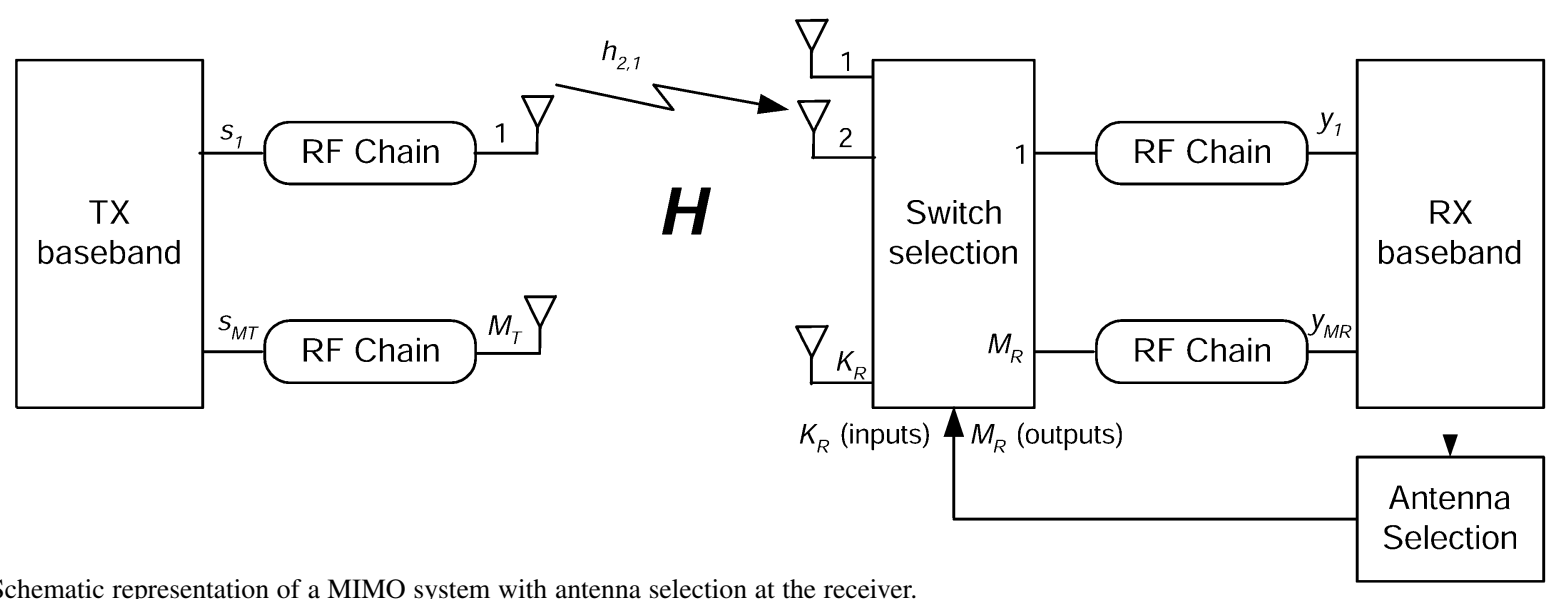

Fig. 1. Schematic representation of a MIMO system with antenna selection at the receiver.

but the performance is close to that of the maximum likelihood (ML) receiver [18]. We develop different antenna-selection criteria assuming that the channel-state information is available at the receiver. Simulations show that antenna selection leads to an improvement of diversity and coding gain with respect to the system without antenna selection and achieves the same diversity as the system using the whole set of available antennas. In addition, we compare the ER achieved using each of the selection criteria and discuss their complexity.

The antenna-selection problem can be formulated as a discrete optimization problem, i.e., find the antenna indices to be used to maximize an objective function (e.g., mutual information). When the channel is perfectly known, the optimization problem becomes an exhaustive search over all possible solutions. However, in real communication systems, the receiver typically has a noisy estimate of the channel. Hence, in this paper we present a globally convergent discrete stochastic approximation algorithm for selecting the optimal antenna subset when the receiver can only evaluate a noisy estimate of the objective function. This algorithm is based on advanced discrete stochastic optimization techniques that appeared in the operations research literature [19].

The remainder of this paper is organized as follows. In Section II, the MIMO system model with traditional linear receivers and antenna selection is presented. In Section III, the LRA receivers are described and in Section IV, several antenna-selection criteria are developed as bounds on the error probability. Simulation examples are provided to demonstrate the improvement in using antenna selection and the performance of different selection criteria. In Section V, a globally convergent adaptive selection algorithm is proposed when only noisy estimates of the channel are available. Section VI contains the conclusion.

\section{SYSTEM DESCRIPTION}

We consider a MIMO system, as shown in Fig. 1, with $M_{T}$ transmit and $M_{R}\left(M_{R} \geq M_{T}\right)$ receive $\mathrm{RF}$ chains and suppose that there are $K_{R} \geq M_{R}$ receive antennas. In this paper, we consider that the antenna selection is implemented only at the receiver. This selection may also be implemented at the transmitter, with the difference that the receiver needs to feedback information about the selected antennas or channel-state infor- mation. The wireless channel is assumed to be quasistatic and flat fading and can be represented by an $\left(K_{R} \times M_{T}\right)$ matrix $\boldsymbol{H}$ whose element $h_{i j}$ represents the complex gain of the channel between the $j$ th transmit antenna and the $i$ th receive antenna. The subset of $M_{R} \leq K_{R}$ receive antennas is determined by the antenna-selection algorithm based on different criteria, which will be developed in the next section. In this paper, we assume that the channel matrix $\boldsymbol{H}$ is known at the receiver, but not at the transmitter.

Denote each of the antenna subsets as $\omega=$ $\left\{\operatorname{Ant}(1), \operatorname{Ant}(2), \ldots, \operatorname{Ant}\left(M_{R}\right)\right\} \quad$ (e.g., selecting the first, second, and sixth antennas is equivalent to $\omega=\{1,2,6\}$ ). Denote the set of all $P=\left(\begin{array}{l}K_{R} \\ M_{R}\end{array}\right)$ possible antenna subsets as $\Omega=\left\{\omega_{1}, \omega_{2}, \ldots, \omega_{P}\right\}$ and denote $\boldsymbol{H}_{\omega}$ as the $\left(M_{R} \times M_{T}\right)$ channel submatrix corresponding to the receive antenna subset $\omega$, i.e., rows of $\boldsymbol{H}$ corresponding to the selected antennas. Then, the aim of antenna-selection algorithms in this paper is to select one of the antenna subsets in $\Omega$ to minimize the ER, e.g., SER or BER. Thus, the optimization problem becomes

$$
\omega^{*}=\arg \min _{\omega \in \Omega} \operatorname{ER}\left(\boldsymbol{H}_{\omega}\right)
$$

where we use $\omega^{*}$ to denote the global minimizer of $\operatorname{ER}\left(\boldsymbol{H}_{\omega}\right)$. In practice, exact expressions of the ER do not usually exist and, therefore, we develop expressions that bound or approximate the ER. As expected, the exact ER will depend on the specific constellation, channel realization, and receiver structure.

The received signal can be expressed as

$$
\boldsymbol{y}=\sqrt{\frac{\rho}{M_{T}}} \boldsymbol{H}_{\omega} \boldsymbol{s}+\boldsymbol{n}
$$

where $\boldsymbol{s}=\left[s_{1}, s_{2}, \ldots, s_{M_{T}}\right]^{T}$ is the $\left(M_{T} \times 1\right)$ transmitted signal vector, $\boldsymbol{y}=\left[y_{1}, y_{2}, \ldots, y_{M_{R}}\right]^{T}$ is the $\left(M_{R} \times 1\right)$ received signal vector, $\boldsymbol{n}$ is the $\left(M_{R} \times 1\right)$ received noise vector, and $\rho$ is the total SNR independent of the number of transmit antennas. The entries of $\boldsymbol{n}$ are independent and identically distributed (i.i.d.) circularly symmetric complex Gaussian variables with unit variance, i.e., $n_{i} \sim \mathcal{N}_{c}(0,1)$. For notational convenience, we do not explicitly specify the dependence of $\boldsymbol{n}$ and $\boldsymbol{y}$ on $\omega$.

\section{A. Traditional Linear Receivers}

Linear receivers are the simplest receivers. In linear receivers, the received signal vector $\boldsymbol{y}$ is linearly transformed by a ma- 


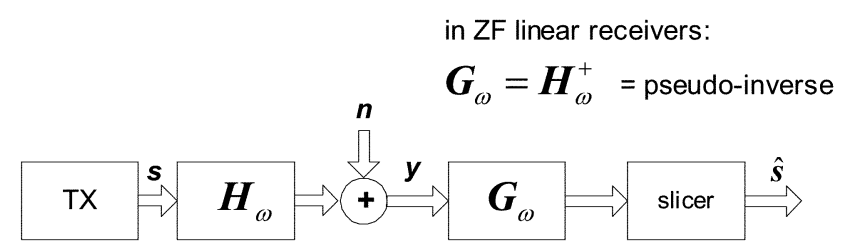

Fig. 2. Traditional linear detector.

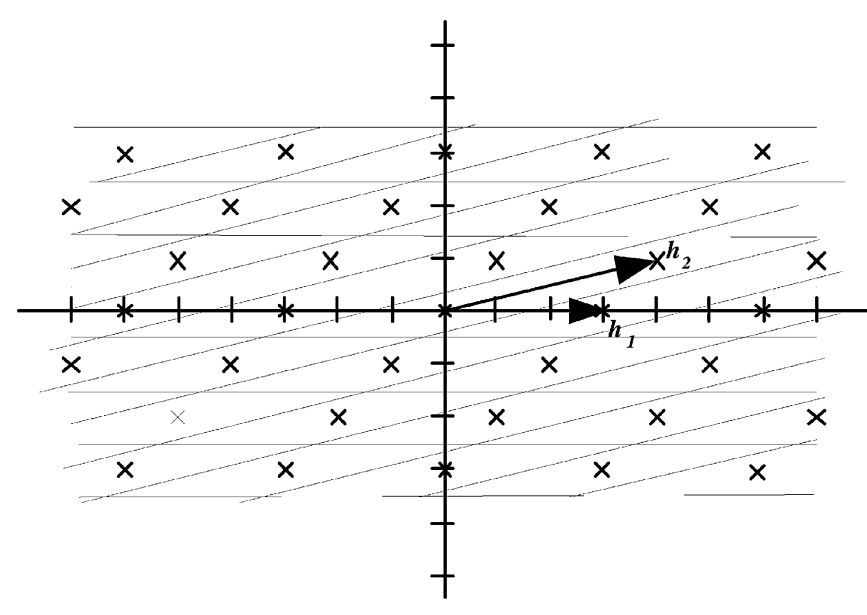

Fig. 3. Received constellation and linear receiver decision regions.

trix equalizer that basically undoes the effects of the channel to obtain

$$
\boldsymbol{r}=\boldsymbol{G}_{\omega} \boldsymbol{y}=\sqrt{\frac{\rho}{M_{T}}} \boldsymbol{G}_{\omega} \boldsymbol{H}_{\omega} \boldsymbol{s}+\boldsymbol{G}_{\omega} \boldsymbol{n}
$$

which is later quantized to obtain an estimate of the transmitted symbol vector $\hat{\boldsymbol{s}}=Q(\boldsymbol{r})$. The whole process is shown in Fig. 2. The matrix equalizer $\boldsymbol{G}_{\omega}$ can be computed according to different criteria. For the zero-forcing (ZF) criterion, the equalizer is given by $\boldsymbol{G}_{\omega}=\sqrt{\left(M_{T}\right) /(\rho)} \boldsymbol{H}_{\omega}^{\dagger}$, where $\boldsymbol{H}_{\omega}^{\dagger}$ denotes the pseudoinverse. The ZF criterion suffers from noise enhancement since it focuses on canceling the effects of the channel response at the expense of enhancing the noise, possibly significantly. In [18], a good explanation of what occurs to the decision regions of the received constellation in a linear receiver is presented. As an example, consider a real-valued MIMO system with $M_{T}=M_{R}=2$, where the transmitted symbols $s_{1}$ and $s_{2}$ belong to a $(2 N+1)$-PAM constellation, i.e., $s_{i} \in\{-N,-N+$ $1, \ldots, 0, \ldots, N-1, N\}$. Assume that the channel matrix is $\boldsymbol{H}_{\omega}=\left[\begin{array}{ll}3 & 4 \\ 0 & 1\end{array}\right]$. Therefore, the received constellation consisting of $\boldsymbol{H}_{\omega} \boldsymbol{s}=s_{1}[3,0]^{T}+s_{2}[4,1]^{T}$ will be a lattice of integer combinations of two vectors, namely $\boldsymbol{h}_{\omega, 1}=[3,0]^{T}$ and $\boldsymbol{h}_{\omega, 2}=$ $[4,1]^{T}$, as shown in Fig. 3. Because of the equalizing operation and the direction of the basis vectors, the decision regions become narrow parallelograms for which small noise samples can make the decoder make a wrong decision.

On the other hand, the minimum mean-square error (mmse) linear equalizer $\boldsymbol{G}_{\omega}=\sigma_{s}^{2} \sqrt{(\rho) /\left(M_{T}\right)} \boldsymbol{H}^{H}\left(\left(\sigma_{s}^{2} \rho\right) /\left(M_{T}\right) \boldsymbol{H} \boldsymbol{H}^{H}+\sigma_{n}^{2} \boldsymbol{I}_{M_{R}}\right)^{-1}$ minimizes the error due to the noise and the interference combined. Other receivers with complexity and performance between the ML and the linear receivers are the BLAST

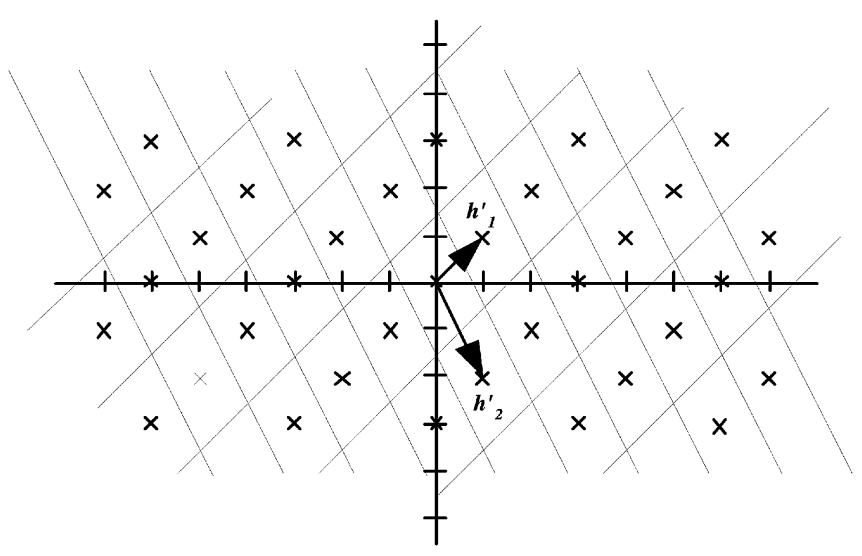

Fig. 4. Decision regions of a linear receiver on the reduced lattice basis.

receivers [20]. Although linear receivers have very low complexity, the performance is far from the more complex optimal ML receivers.

\section{LATTICE-AIDED-REDUCTION LINEAR RECEIVERS}

As shown in Fig. 3, the problem with linear receivers is that the decision regions are very narrow when the basis of the lattice are highly correlated. A solution is to find a different basis for the same lattice for which the decision regions are more robust against the noise. Then, the equalizing operation is performed in this new basis for which wrong decisions are more unlikely to occur and, finally, the decoded symbols are transformed to the original basis. In this section, we describe how the change of basis can be optimized to improve the decision regions in linear receivers and how the receiver can make use of this improvement. For notational convenience, we will drop the subscript $\omega$.

\section{A. Constellations, Lattices, and Basis Change}

An $M_{T}$-dimensional lattice, though infinite, can be described in terms of a generator basis $\left\{\boldsymbol{h}_{1}, \ldots, \boldsymbol{h}_{M_{T}}\right\}$. Associated to them, there is a generator matrix $\boldsymbol{H}$ whose columns are $\left\{\boldsymbol{h}_{1}, \ldots, \boldsymbol{h}_{M_{T}}\right\}$ and the lattice is described as integer (possibly complex) combinations of the generators as

$$
\begin{aligned}
L\left(\boldsymbol{h}_{1}, \boldsymbol{h}_{2}, \ldots, \boldsymbol{h}_{M_{T}}\right) & =\sum_{i=1}^{M_{T}} \mathbb{Z}_{\mathbb{C}} \boldsymbol{h}_{i} \\
& =\left\{\sum_{i=1}^{M_{T}} s_{i} \boldsymbol{h}_{i}, s_{i} \in \mathbb{Z}_{\mathbb{C}}=\mathbb{Z}+j \mathbb{Z}\right\} .
\end{aligned}
$$

If $\boldsymbol{H}$ is a basis of a lattice, $\boldsymbol{H}^{\prime}=\boldsymbol{H P}$ also is a basis of the same lattice if $\boldsymbol{P}$ and $\boldsymbol{P}^{-1}$ have integer (possibly complex) entries. Therefore, the aim of the LRA receivers is to find a change of basis $\boldsymbol{P}$ that transforms the generators into $\boldsymbol{H}^{\prime}$ to optimize the decision regions for an specific lattice and decoder. This problem is known as the lattice-reduction problem. The goal of lattice basis reduction is, given an arbitrary lattice basis, obtain a basis of the shortest possible vectors; that is, vectors as close as possible to mutually orthogonal. If we consider the lattice in Fig. 3, a possible new basis $\boldsymbol{h}_{1}^{\prime}$ and $\boldsymbol{h}_{2}^{\prime}$ for the same lattice is the one shown in Fig. 4. The generators of this basis are close to orthogonal and, therefore, the decision regions are improved as can be observed comparing both figures. 


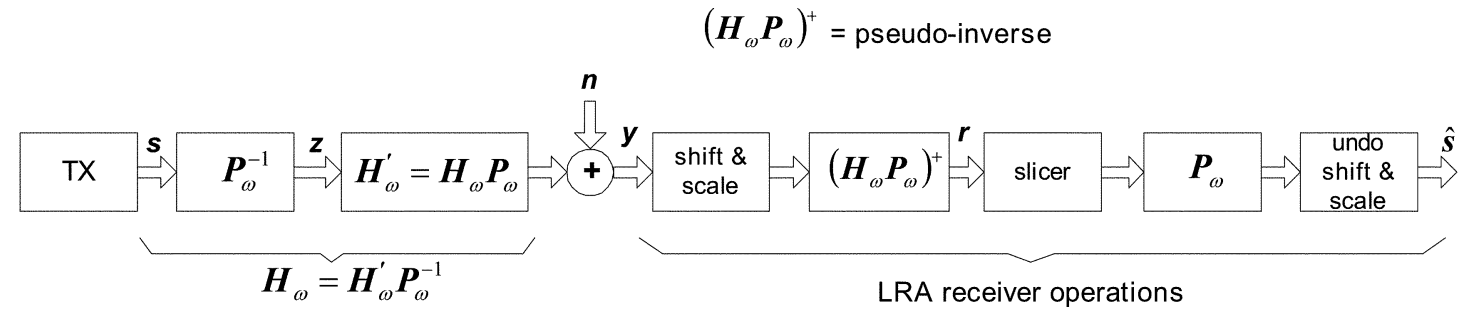

Fig. 5. Linear detector using lattice reduction for a general QAM constellation.

\section{B. Receiver Operations}

The idea behind the LRA receivers is to operate in the more appropriate reduced basis. An input symbol vector represented by $\boldsymbol{s}$ in the original basis with elements in $\mathbb{Z}_{\mathbb{C}}$ can be represented by $\boldsymbol{z}=\boldsymbol{P}^{-1} \boldsymbol{s}$ in the reduced basis. Note that since $\boldsymbol{P}^{-1}$ has entries restricted to $\mathbb{Z}_{\mathbb{C}}$, then $z$ also has elements in $\mathbb{Z}_{\mathbb{C}}$. The LRA receiver uses the reduced basis, since the decision regions are improved and, then, it returns the decoded symbols to the original basis. We can assume that the received vector $\boldsymbol{y}$ in (2) is already in the reduced basis, i.e.,

$$
\boldsymbol{y}=\sqrt{\frac{\rho}{M_{T}}} \boldsymbol{H}\left(\boldsymbol{P} \boldsymbol{P}^{-1}\right) \boldsymbol{s}+\boldsymbol{n}=\sqrt{\frac{\rho}{M_{T}}} \boldsymbol{H}^{\prime} \boldsymbol{z}+\boldsymbol{n} .
$$

In particular, for the linear receiver, the matrix equalizer $\boldsymbol{G}$ will compensate for the effects of the channel in the reduced basis, i.e., $\boldsymbol{H}^{\prime}=\boldsymbol{H} \boldsymbol{P}$. For the $\mathrm{ZF}$ receiver $\boldsymbol{G}=\sqrt{\left(M_{T}\right) /(\rho)}(\boldsymbol{H} \boldsymbol{P})^{\dagger}$, obtaining a decision statistic of $z$ as

$$
\boldsymbol{r}=\sqrt{\frac{\rho}{M_{T}}} \boldsymbol{G}(\boldsymbol{H P}) \boldsymbol{z}+\boldsymbol{G n}
$$

which is quantized to obtain an estimate of $z$, i.e., $\hat{z}=Q(\boldsymbol{r})$. Since the lattice points consist of elements in $\mathbb{Z}_{\mathbb{C}}$, the quantization consists of a simple rounding operation in which real and imaginary parts are rounded separately. Then, an estimate of the transmitted vector $s$ is obtained, returning to the original basis as $\hat{\boldsymbol{s}}=\boldsymbol{P} \hat{\boldsymbol{z}}$. Note that the process can be implemented as long as the original symbols $s_{i}$ belong to $\mathbb{Z}_{\mathbb{C}}$.

To make use of the lattice theory, original symbols in the constellation $s_{i}$ are required to consist of symbols in $\mathbb{Z}_{\mathbb{C}}$, as expressed in (4). Note that the origin $[0, \ldots, 0]^{T}$ also belongs to the lattice. Common quadrature amplitude modulation (QAM) constellations neither consist of contiguous integers nor contain the origin; hence, a scaling and shifting of the whole constellation is necessary. As an example, consider 16-QAM symbols, such that $\Re\left\{s_{i}\right\} \in\{-3,-1,1,3\}$ and $\Im\left\{s_{i}\right\} \in\{-3,-1,1,3\}$. A possible lattice satisfying our requirement might be constructed shifting each symbol by $d_{i}=1+i$ and scaling by $1 / 2$. In this way, the QAM symbol vector becomes $\boldsymbol{s}^{\prime}=(1 / 2)(\boldsymbol{s}+\boldsymbol{d})$, i.e., $\Re\left\{s_{i}^{\prime}\right\} \in\{-1,0,1,2\}$ and $\Im\left\{s_{i}^{\prime}\right\} \in\{-1,0,1,2\}$. Therefore, a basis change with $\boldsymbol{P}$ consisting of entries $\mathbb{Z}_{\mathbb{C}}$ converts lattice points into other points in the same lattice, with the difference that the new lattice is now treated as infinite, since entries in $\boldsymbol{P}$ do not have any size constraint. To make LRA receivers general to any spatial multiplexing transmitter with standard QAM constellations and assuming that the channel matrix $\boldsymbol{H}$ is known at the receiver, the scaling and shifting operation should be implemented at the receiver. As an example, consider the $\left(M_{T} \times 1\right)$ shifting vector $\boldsymbol{d}=[1+i, \ldots, 1+i]^{T}$. Then, assuming that the shifted and scaled constellation was transmitted, the received vector becomes

$$
\boldsymbol{y}^{\prime}=\sqrt{\frac{\rho}{M_{T}}} \boldsymbol{H} \boldsymbol{s}^{\prime}=\sqrt{\frac{\rho}{M_{T}}} \boldsymbol{H} \frac{1}{2}[\boldsymbol{s}+\boldsymbol{d}] .
$$

Considering that $\boldsymbol{y}$ is the signal reaching the receiver and was transmitted using the original symbol vector $\boldsymbol{s}$, the signal in (7) can be obtained at the receiver as

$$
\boldsymbol{y}^{\prime}=\frac{1}{2} \boldsymbol{y}+\frac{1}{2} \sqrt{\frac{\rho}{M_{T}}} \boldsymbol{H} \boldsymbol{d} .
$$

Then, the operations at the receiver become scale, shift, and equalize in the new basis

$$
\begin{aligned}
\boldsymbol{r}= & \underbrace{\sqrt{\frac{M_{T}}{\rho}}(\boldsymbol{H P})^{\dagger}}_{\text {equalize in new basis }} \underbrace{\frac{1}{2}}_{\text {scale }} \\
& {[\underbrace{\sqrt{\frac{\rho}{M_{T}} \boldsymbol{H P}\left(\boldsymbol{P}^{-1} \boldsymbol{s}\right)+\boldsymbol{n}}+\underbrace{\sqrt{\frac{\rho}{M_{T}}}(\boldsymbol{H P})\left(\boldsymbol{P}^{-1} \boldsymbol{d}\right)}_{\text {shift }}] .}_{\text {rx signal-y }} .}
\end{aligned}
$$

In particular, when $M_{T}=M_{R}$ and the channel matrix is full rank, we have $(\boldsymbol{H P})^{\dagger}=\boldsymbol{P}^{-1} \boldsymbol{H}^{-1}$. Equation (9) can be rewritten as

$$
\boldsymbol{r}=\boldsymbol{P}^{-1} \boldsymbol{s}^{\prime}+\frac{1}{2} \sqrt{\frac{M_{T}}{\rho}}(\boldsymbol{H P})^{\dagger} \boldsymbol{n} .
$$

Hence, the second step at the receiver becomes slice, return to original basis, and undo the scale and shift

$$
\hat{\boldsymbol{s}}=2 \boldsymbol{P} Q(\boldsymbol{r})-\boldsymbol{d} .
$$

Note that the slicer $Q(\cdot)$ is just a rounding operation, since the symbols in the lattice belong to $\mathbb{Z}_{\mathbb{C}}$. The whole process described in (9) and (11) is shown in Fig. 5.

\section{Basis Reduction}

So far, we have assumed that, given a basis for the lattice, a reduced basis can be obtained, e.g., given a lattice as the one shown in Fig. 3, we can obtain a reduced basis for the same lattice as the one shown in Fig. 4. We now give an overview of the Gauss basis reduction algorithm limited to rank $M_{T}=2$, which is used in [18]. The reduction algorithm uses a method similar to the Gram-Schmidt orthogonalization. Assume that $\boldsymbol{h}_{1}$ and $\boldsymbol{h}_{2}$ are a basis of the lattice. Define the Euclidean inner 


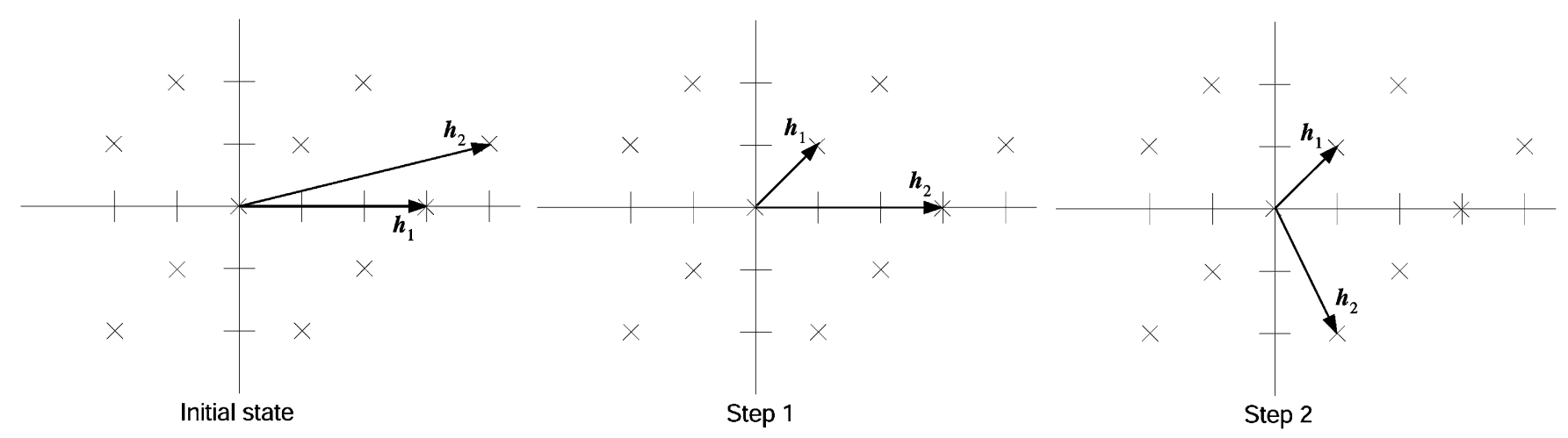

Fig. 6. Lattice basis reduction of lattice in Fig. 3 using Algorithm 1.

product as $\left\langle\boldsymbol{h}_{1}, \boldsymbol{h}_{2}\right\rangle=\boldsymbol{h}_{1}^{H} \boldsymbol{h}_{2}$ and consider the Euclidean norm. Assuming that $\left\|\boldsymbol{h}_{1}\right\|<\left\|\boldsymbol{h}_{2}\right\|$, the basis reduction algorithm does operations in the basis vectors of the form

$$
\boldsymbol{h}_{2}=\boldsymbol{h}_{1}-\mu \boldsymbol{h}_{2}
$$

which yields a different basis for the same lattice if $\mu \in \mathbb{Z}_{\mathbb{C}}$. Since the purpose of the lattice reduction is to make lattice basis vectors as close to orthogonal as possible, Gram-Schmidt orthogonalization can be used to find $\mu$ with the further constraint $\mu \in \mathbb{Z}_{\mathbb{C}}$. The ideal Gram-Schmidt orthogonalization uses $\mu^{\prime}=\left(\left\langle\boldsymbol{h}_{1}, \boldsymbol{h}_{2}\right\rangle\right) /\left(\left\langle\boldsymbol{h}_{1}, \boldsymbol{h}_{2}\right\rangle\right)$, but this operation would change the lattice, since $\mu^{\prime}$ is not in $\mathbb{Z}_{\mathbb{C}}$. The weakly reduced Gram-Schmidt orthogonalization uses an integer rounding of the ideal Gram-Schmidt coefficient as $\mu=\left\lfloor\mu^{\prime}\right\rceil$, where real and imaginary parts of complex numbers are rounded separately. Using a weak reduction, the lattice remains the same. Once $\boldsymbol{h}_{2}$ has been reduced with respect to $\boldsymbol{h}_{1}$, if $\boldsymbol{h}_{2}<\boldsymbol{h}_{1}$ we have the possibility of reducing $\boldsymbol{h}_{1}$ with respect to the new $\boldsymbol{h}_{2}$. We first swap $\boldsymbol{h}_{1}$ and $\boldsymbol{h}_{2}$. This second reduction will occur if such $\mu$ exists, i.e., if $\left|\Re\left\{\left\langle\boldsymbol{h}_{1}, \boldsymbol{h}_{2}\right\rangle\right\}\right|>(1 / 2)\left\|\boldsymbol{h}_{1}\right\|^{2}$ or $\left|\Im\left\{\left\langle\boldsymbol{h}_{1}, \boldsymbol{h}_{2}\right\rangle\right\}\right|>(1 / 2)\left\|\boldsymbol{h}_{1}\right\|^{2}$. The algorithm repeats this process until no more reduction is possible. As an example, given the basis in Fig. 3, the steps performed in the algorithm are represented in Fig. 6.

A notion of lattice reduction for a lattice basis $\boldsymbol{h}_{1}, \ldots, \boldsymbol{h}_{M_{T}}$ of arbitrary rank $M_{T}$ was proposed by Lenstra, Lenstra, and Lovasz (LLL) in [21]. It uses ideas similar to the Gauss reduction algorithm. For a given $\delta,(1 / 4)<\delta<1$, the LLL reduction algorithm modifies an input basis $\boldsymbol{h}_{1}, \ldots, \boldsymbol{h}_{M_{T}}$ so that the output basis satisfies the following $\delta$-reduction properties:

and

$$
\mu_{i, j} \leq \frac{1}{2} \quad \text { for } \quad 1 \leq j<i \leq M_{T}
$$

$$
\delta \cdot\left\|\hat{\boldsymbol{h}}_{k-1}\right\|^{2}>\left\|\hat{\boldsymbol{h}}_{k}+\mu_{k, k-1} \hat{\boldsymbol{h}}_{k-1}\right\|^{2}
$$

where the vectors $\hat{\boldsymbol{h}}_{1}, \ldots, \hat{\boldsymbol{h}}_{M_{T}}$ denote the Gram-Schmidt orthogonalization of the output basis that can be obtained by the recursion

$$
\begin{aligned}
& \hat{\boldsymbol{h}}_{1}=\boldsymbol{h}_{1} \\
& \hat{\boldsymbol{h}}_{i}=\boldsymbol{h}_{i}-\sum_{j=1}^{i-1} \mu_{i, j} \hat{\boldsymbol{h}}_{j}, \quad \text { for } \quad i=2, \ldots, M_{T}
\end{aligned}
$$

and the Gram-Schmidt coefficients are equal to

$$
\mu_{i, j}=\frac{\left\langle\hat{\boldsymbol{h}}_{j}, \boldsymbol{h}_{i}\right\rangle}{\left\langle\hat{\boldsymbol{h}}_{j}, \hat{\boldsymbol{h}}_{j}\right\rangle} .
$$

A possible implementation of the LLL algorithm to obtain the reduced basis is given in Algorithm 1.

Algorithm 1 LLL lattice-reduction algorithm

INPUT: Lattice basis $\boldsymbol{h}_{1}=\boldsymbol{H}[:, 1], \ldots, \boldsymbol{h}_{M_{T}}=\boldsymbol{H}\left[:, M_{T}\right] \in$ $\mathbb{C}^{M_{T}}$ and $(1 / 4)<\delta<1 \quad k=2$

WHILE $k \leq M_{T}$ DO

FOR $i=k-1, \ldots, 1 \mathrm{DO}$

$\boldsymbol{h}_{k}=\boldsymbol{h}_{k}-\left\lfloor\mu_{k, i}\right\rceil \boldsymbol{h}_{i} ;$ round real and image separately

END FOR

Compute $\hat{\boldsymbol{h}}_{k}$ as in (15)

IF $\delta\left\|\hat{\boldsymbol{h}}_{k-1}\right\|^{2}>\left\|\hat{\boldsymbol{h}}_{k}+\mu_{k, k-1} \hat{\boldsymbol{h}}_{k-1}\right\|^{2}$ THEN

$\boldsymbol{h}_{k-1} \leftrightarrow \boldsymbol{h}_{k}$ (exchange)

$k=\max (k-1,2)$

ELSE $k=k+1$

END WHILE

OUTPUT: Reduced lattice basis $\boldsymbol{H}^{\prime}=\left[\boldsymbol{h}_{1}, \ldots, \boldsymbol{h}_{M_{T}}\right]$ and $\boldsymbol{P}$ defined as $\boldsymbol{H}^{\prime}=\boldsymbol{H} \boldsymbol{P}$

During the algorithm, we keep two sets of vectors, namely the lattice reduced basis vectors $\left\{\boldsymbol{h}_{1}, \ldots, \boldsymbol{h}_{k}\right\}$ and the Gram-Schmidt vectors $\left\{\hat{\boldsymbol{h}}_{1}, \ldots, \hat{\boldsymbol{h}}_{k}\right\}$ (with the corresponding Gram-Schmidt coefficients $\mu_{i, j}$ ), which are continuously updated. Note that only adjacent vectors $\boldsymbol{h}_{k-1}$ and $\boldsymbol{h}_{k}$ may be exchanged. When the rank is $M_{T}=2$ and $\delta=1$, Algorithm 1 is identical to the Gauss reduction algorithm used in [18]. Reduced bases with better properties can be obtained when the constant $\delta$ is closer to one, although the number of iterations of Algorithm 1 would increase. More efficient implementations of the algorithm can be found in [22] and [23].

Other types of reduced bases are the Korkin-Zolotarev (KZ) basis [24]-[26], the Minkowski basis [27], [28], the Seysen basis [29], [30], and hybrids [31], which have different reduction criteria. These bases have, in general, slightly better properties (they are usually LLL reduced too), although the reduction is more time consuming.

The performance of the LRA receivers will be closer to that of the ML receiver as the size of the QAM constellation increases. This occurs because LRA treats finite constellations as 
infinite and, therefore, constellation points on the boundary of the constellation that originally had less constellation neighbors end up having the same number of neighbors as the internal constellation points. Hence, this loss in performance will be smaller if the ratio of boundary constellation points and internal points becomes smaller, which occurs in high-order QAM constellations (e.g., 64 or 256 QAM). Moreover, it is known that the computational complexity of the ML decoder in MIMO systems with large constellations or large number of transmit antennas becomes prohibitive. Therefore, LRA decoders are a good alternative when large-order constellations or a large number of transmit antennas are used. Note that the complexity of the LRA receivers has two parts: 1) computing the reduced basis of the lattice and 2) implementing the linear equalizer. In quasistatic channels, the lattice is fixed during a long period of time, so the basis reduction is performed just once and then the resulting basis is stored for subsequent use. Thus, the complexity of solving 1) is not of major concern.

\section{ANTENNA-SELECTION CRITERIA}

In this section, we develop different antenna-selection criteria to select an optimal antenna subset in LRA linear receivers when the channel matrix $\boldsymbol{H}$ is known at the receiver.

\section{A. Maximum MIMO Mutual Information}

Assuming that the MIMO channel $\boldsymbol{H}_{\omega}$ is known at the receiver but not at the transmitter, the mutual information between the transmitter and receiver is given by [2]

$$
I_{\omega}=\log \operatorname{det}\left(\boldsymbol{I}_{M_{T}}+\frac{\rho}{M_{T}} \boldsymbol{H}_{\omega}^{H} \boldsymbol{H}_{\omega}\right) .
$$

Then, one criterion to select the optimal antenna is to select the antenna subset $\omega$, which maximizes the above mutual information [3]

$$
\omega_{I}^{*}=\arg \max _{\omega \in \Omega}\left(I_{\omega}\right)
$$

In [4], a suboptimal algorithm is presented to reduce the complexity when the number of antenna subsets in $\Omega$ is high. This method considers a recursive algorithm that successively eliminates one receive antenna, i.e., one row of $\boldsymbol{H}$, until there are $M_{R}$ antennas left. The loss performance of the suboptimal algorithm is minimum.

\section{B. Largest Minimum Eigenmode}

As shown in [2], MIMO systems can be described in terms of spatial eigenmodes that act as parallel SISO subchannels. The SNR of each eigenmode is proportional to each of the singular values of the MIMO channel $\boldsymbol{H}_{\omega}$. It is expected that the ER will be influenced by the worst eigenmode of the channel. Define the singular values of $\boldsymbol{H}_{\omega}$ as $\left(\lambda_{\omega}\right)_{i}\left(i=1, \ldots, \min \left(M_{T}, M_{R}\right)\right)$. Therefore, an antenna-selection criterion can be simplified to [11]

$$
\omega_{\text {eig }}^{*}=\underset{\omega \in \Omega}{\arg \max }\left\{\min _{i \leq M_{T}}\left(\lambda_{\omega}\right)_{i}\right\}
$$

Note that the two first criteria do not need to perform the lattice basis reduction.

\section{Minimum ER by Simulation}

The maximum mutual information criterion can minimize the error probability when the receiver has infinite complexity. For finite complexity receivers, the maximum mutual information criterion does not necessarily minimize the ER that depends on the specific receiver operations. However, the ER cannot be expressed in closed form. Since it is difficult to find exact BER or SER expressions in MIMO receivers; when the number of different antenna subsets is not very high, we could just run simulations at the receiver with fake symbols to find the optimal antenna subset. Assume that the channel $\boldsymbol{H}_{\omega}$ and SNR $\rho$ are known at the receiver. At the receiver, generate $m$ fake random symbol vectors $\boldsymbol{S}_{f}=\left[\boldsymbol{s}_{f}(1), \ldots, \boldsymbol{s}_{f}(m)\right]$ with $s_{f, k}(i)$ belonging to the original transmitted constellation. Each $\omega \in \Omega$ perform a simulation of the form

$$
\boldsymbol{Y}_{f}=\sqrt{\frac{\rho}{M_{T}}} \boldsymbol{H}_{\omega} \boldsymbol{S}_{f}+\boldsymbol{N}
$$

where the $\left(M_{R} \times m\right)$ matrix $\boldsymbol{N}$ contains i.i.d. $\mathcal{N}_{c}(0,1)$ samples. Note that the symbols are not sent through the channel; they are simply generated with a computer simulation. At the receiver, perform the LRA detection operations in (9) and (11) to obtain $\widehat{\boldsymbol{S}}_{f}$ and estimate the $\widehat{\mathrm{SER}}_{\omega}$ or $\widehat{\mathrm{BER}}_{\omega}$ comparing $\boldsymbol{S}_{f}$ and $\hat{\boldsymbol{S}}_{f}$. Hence, a criterion to select an antenna subset that minimizes the ER becomes

$$
\omega_{\mathrm{sim}}^{*}=\arg \min _{\omega \in \Omega}\left\{\widehat{\operatorname{SER}}_{\omega}\right\} .
$$

The disadvantage of the method is that in the high SNR regime, SER can be very low and, therefore, a large amount of fake symbols need to be sent if we want to obtain a good estimate of the SER.

On the other hand, it has been observed by simulations that the antenna subset having the minimum ER at high SNR values corresponds to the antenna subset having the minimum ER at lower SNR (as long as there is not a large SNR difference). Therefore, to find the best antenna subset when the SNR is high, we can reduce the SNR for the simulation and less fake symbols may be needed to obtain a good estimate of the ER.

We note that although this approach is highly complex, it could be used in other MIMO systems for which tight bounds on the ER are not easy to find because of the specific receiver or because of the channel model or noise model. Note that in static channels, antenna selection needs to be performed only once. Hence, a receiver could afford this complexity if an improvement in the performance can be obtained. Moreover, the method can be used to minimize the bit, symbol or frame ER.

\section{D. mse}

In linear receivers, the error probability of each data stream is influenced by the mean-square error (mse) between the symbol $z_{i}^{\prime}$ and its estimate $r_{i}$ prior to being sliced. From (10) we have

$$
\boldsymbol{r}=\boldsymbol{z}^{\prime}+\frac{1}{2} \sqrt{\frac{M_{T}}{\rho}}\left(\boldsymbol{P} \boldsymbol{H}_{\omega}\right)^{\dagger} \boldsymbol{n}=\boldsymbol{z}^{\prime}+\frac{1}{2} \boldsymbol{G}_{\omega} \boldsymbol{n}
$$


and, therefore, the mse of each of the data streams in the new basis becomes

$$
\operatorname{mse}_{\omega, i}=E\left\{\left(z_{i}{ }^{\prime}-r_{i}\right)\left(z_{i}{ }^{\prime}-r_{i}\right)^{*}\right\}=\frac{1}{4}\left\|\boldsymbol{G}_{\omega}[i,:]\right\|^{2}, 1 \leq i \leq M_{T}
$$

where $\boldsymbol{G}_{\omega}[i,:]$ denotes the $i$ th row of $\boldsymbol{G}_{\omega}$. Then, the ER will be influenced by the data stream with maximum mse. Thus, a selection criterion can be simplified to select the antenna subset with minimum maximum mse

$$
\omega_{\text {mse }}^{*}=\arg \min _{\omega \in \Omega}\left\{\max _{i \leq M_{T}} \mathrm{mse}_{\omega, i}\right\} .
$$

1) Error Propagation: In the LRA receiver, after the slicing operation the symbols are transformed to the original basis as $\boldsymbol{s}=\boldsymbol{P \boldsymbol { s } ^ { \prime }}$. Then, if an error has occurred in the reduced basis in the $i$ th position, it will propagate to as many symbols as nonzero elements exist in the $i$ th column of $\boldsymbol{P}$. Consider two antenna subsets having similar maximum mse. Then, between these two subsets, we would like to choose the one that does not propagate the error (or, at least, it propagates to a lesser number of symbols). To quantify the error propagation, we approximate the symbol ER at the $i$ th position. If $\boldsymbol{r}=z^{\prime}+(1 / 2) \boldsymbol{G}_{\omega} \boldsymbol{n}=\boldsymbol{z}^{\prime}+\boldsymbol{v}$, then the distance between constellation points after the equalizer operation at the receiver is the same as the distance between constellation points $z_{i}^{\prime}$ at the transmitter. The noise $v$ is Gaussian since it is a linear combination of Gaussian random variables. Further, it is circularly symmetric since it is a linear combination of a circularly symmetric Gaussian vector, i.e., $v_{i} \sim$ $\mathcal{N}_{c}\left(0\right.$, mse $\left._{\omega, i}\right)$. Since the data symbols in $\boldsymbol{z}^{\prime}$ belong to $\mathbb{Z}_{\mathbb{C}}$, then the minimum distance between any two constellation points is $d_{\min }=1$. Then, for high SNR, the SER corresponding to the $i$ th transmit antenna can be approximated by

$$
P_{\omega, i} \simeq 2 \cdot \operatorname{Pr}\left(\Re\left\{v_{i}\right\}>\frac{d_{\min }}{2}\right)
$$

with $\Re\left\{v_{i}\right\} \sim \mathcal{N}\left(0,\left(\operatorname{mse}_{\omega, i}\right) /(2)\right)$. Hence

$$
P_{\omega, i} \simeq 2 \cdot Q\left(\frac{d_{\text {min }}}{2 \sqrt{\frac{\mathrm{mse}_{\omega, i}}{2}}}\right)=2 \cdot Q\left(\frac{1}{\sqrt{2 \mathrm{mse}_{\omega, i}}}\right)
$$

where $Q(\cdot)$ is the integral of the tail of the unit variance Gaussian distribution. Denote $k_{i}$ as the number of nonzero elements in the $i$ th column of $\boldsymbol{P}$, i.e., $k_{i}=\operatorname{nnz}(\boldsymbol{P}[:, i])$. Then, the selection criterion becomes

$$
\omega_{\mathrm{mse}-p}^{*}=\arg \min _{\omega \in \Omega}\left\{\max _{i \leq M_{T}} k_{i} \cdot Q\left(\frac{1}{\sqrt{2 \mathrm{mse}_{\omega, i}}}\right)\right\} .
$$

It is expected that this last criterion will improve the performance when the number of rows of $\boldsymbol{P}$ (i.e., the number of transmit antennas $M_{T}$ ) is large.

\section{E. Optimal Geometrical Decision Region}

Since the improvement of LRA detectors comes from optimizing the decision regions, it would be interesting to see the selection criterion from a geometrical perspective. As illustrated

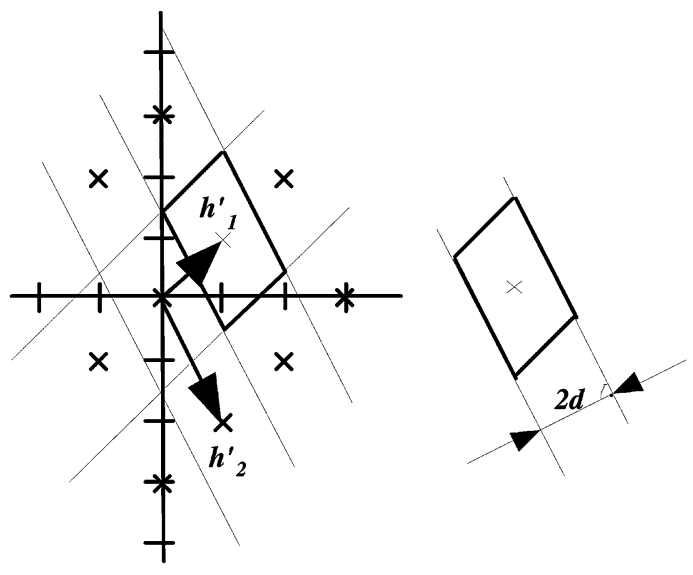

Fig. 7. Decision region in the LRA linear receiver and minimum noise level $\boldsymbol{d}$ required to make a wrong decision.

in Fig. 4 in a real case with $M_{T}=2$, the decision boundaries are defined by the reduced lattice basis. The corresponding decision region is shown in Fig. 7 and is equivalent to a parallelepiped decision region spanned by lattice basis vectors around each lattice point in the center of the region. The minimum value of the noise that provokes a wrong decision is shown in this figure as $\boldsymbol{d}$ and is influenced by both the length of the shortest vector in the reduced lattice basis and the relative angle between the two basis vectors. Note that if the basis vectors are mutually orthogonal, the minimum noise vector that produces an error is clearly the shortest vector in the lattice basis. Since these vectors are not orthogonal, this value can be quantified by orthogonalizing the shortest vector in the reduced lattice basis with respect to the other one and computing its length.

We want to generalize this notion to the more general $\left(M_{R} \times M_{T}\right)$ case. Define the orthogonal projection operator $\pi_{i}\left(\boldsymbol{h}_{i}\right)$ of a vector $\boldsymbol{h}_{i}$ with respect to the subspace $V_{i}=\operatorname{span}\left\{\left\{\boldsymbol{h}_{1}, \ldots, \boldsymbol{h}_{M_{T}}\right\} \backslash \boldsymbol{h}_{i}\right\}$ as

$$
\pi_{i}\left(\boldsymbol{h}_{i}\right)=\boldsymbol{h}_{i}-\operatorname{proj}_{V_{i}} \boldsymbol{h}_{i}
$$

where $\operatorname{proj}_{V_{i}} \boldsymbol{h}_{i}$ can be easily obtained by finding a Gram-Schmidt basis of $V_{i}$ and projecting $\boldsymbol{h}_{i}$ onto it. The most probable error in the symbol transmitted from the $i$ th transmit antenna will occur if the noise level in the direction of $\pi_{i}\left(\boldsymbol{h}_{i}\right)$ is larger than half its length. Define $\boldsymbol{d}_{i}=(1 / 2) \pi_{i}\left(\boldsymbol{h}_{i}\right)$. Then, an antenna-selection criterion can be simplified to selecting the antenna subset with the largest $\left\|\boldsymbol{d}_{i}\right\|$. Then, denoting $\boldsymbol{h}_{\omega, i}^{\prime}$ as the $i$ th column of $\boldsymbol{H}_{\omega}^{\prime}$, the geometrical selection criterion becomes

$$
\begin{aligned}
\omega_{g}^{*} & =\arg \max _{\omega \in \Omega}\left\{\min _{1 \leq i \leq M_{T}}\left\|\boldsymbol{d}_{\omega, i}\right\|\right\} \\
& =\arg \max _{\omega \in \Omega}\left\{\min _{1 \leq i \leq M_{T}} \frac{1}{2}\left\|\pi_{i}\left(\boldsymbol{h}_{\omega, i}^{\prime}\right)\right\|\right\}
\end{aligned}
$$

where $\pi_{i}\left(\boldsymbol{h}_{\omega, i}^{\prime}\right)=\left(\boldsymbol{h}_{\omega, i}^{\prime}-\operatorname{proj}_{V_{i}} \boldsymbol{h}_{\omega, i}^{\prime}\right) \quad$ and $V_{i}=\operatorname{span}\left\{\left\{\boldsymbol{h}_{\omega, 1}^{\prime}, \ldots, \boldsymbol{h}_{\omega, M_{T}}^{\prime}\right\} \backslash \boldsymbol{h}_{\omega, i}^{\prime}\right\}$.

Note that the optimal decision region criterion and the mse criterion are two different ways of looking at the same metric. In the optimal decision region criterion, we view the system before applying the equalizer. In the mse criterion, we view the 
$(2 \times 2)$

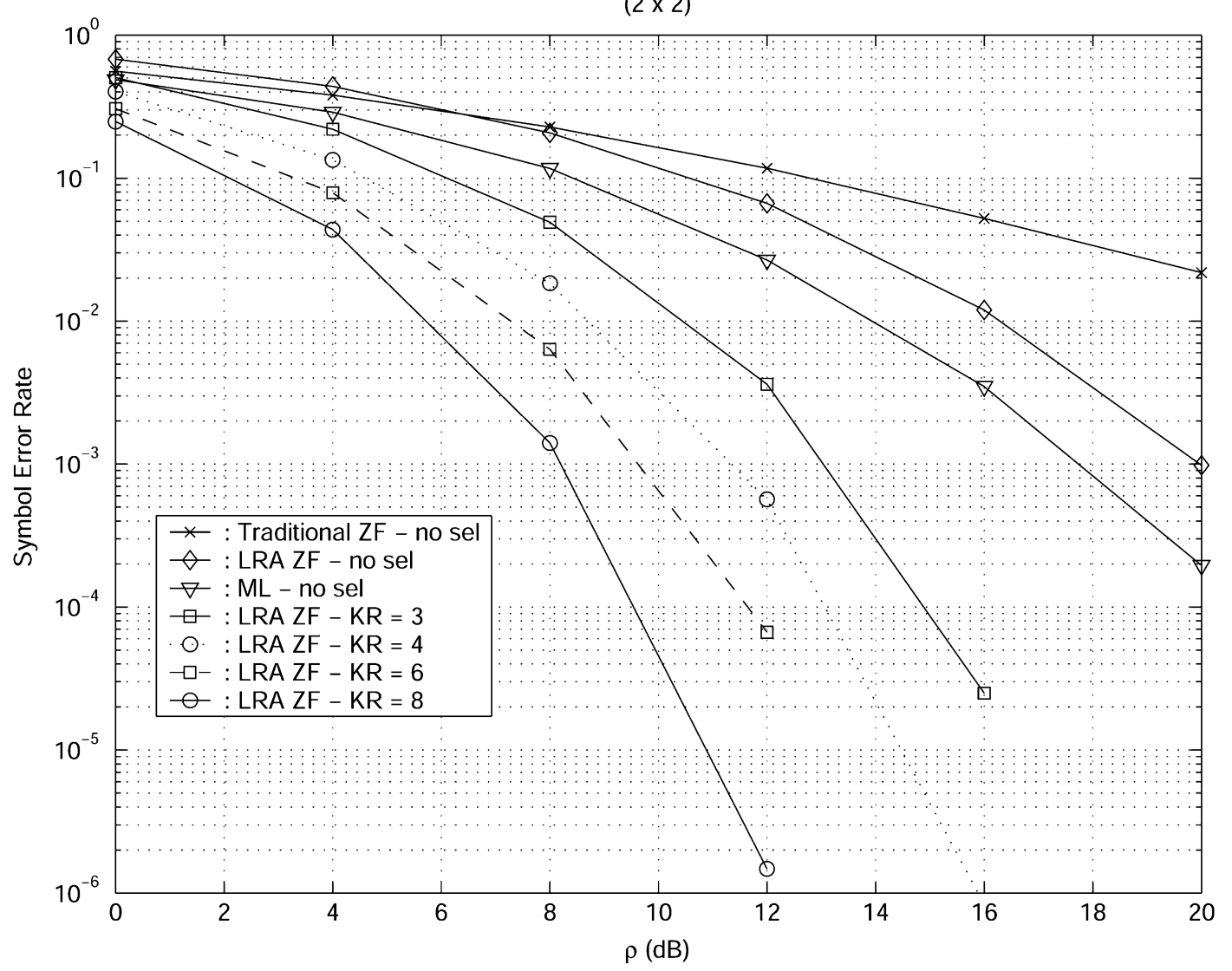

Fig. 8. Error performance comparison among different receivers with and without antenna selection.

system after the equalization has been performed. Therefore, the performance of both criteria will be the same.

1) Error Propagation: Similar to the mse criterion, we would like to select an antenna subset that not only optimizes the lattice reduced decision region, but also minimizes the error propagation. The noise vector before applying the equalizer is circularly symmetric in each dimension with all the dimensions orthogonal. The most probable error will occur if the noise in the direction of $\boldsymbol{d}_{\omega, i}$ is greater than the absolute value of it. Then, we can rotate all the axes so one of the components of the noise is aligned with $\boldsymbol{d}_{\omega, i}$ with variance $\sigma_{n}^{2} / 2$. Therefore, the probability of error in that direction can be approximated by

$$
\operatorname{Pr}\left\{\Re\left\{n_{i}\right\}>\left\|\boldsymbol{d}_{\omega, i}\right\|\right\}=Q\left(\frac{\sqrt{2}\left\|\boldsymbol{d}_{\omega, i}\right\|}{\sigma_{n}}\right)
$$

where we have applied that $\Re\left\{n_{i}\right\} \sim \mathcal{N}\left(0, \sigma_{n}^{2} / 2\right)$. The correct scaling factor in $\boldsymbol{H}$ in the computation of $\boldsymbol{d}_{\omega, i}$ needs to be considered, i.e., consider vectors scaled as $\sqrt{(\rho) /\left(M_{T}\right)} \boldsymbol{H}$ and the original separation between constellation symbols (e.g., $d_{\min }=2$ in the QAM case considered in this paper). As before, denote $k_{i}$ as the number of nonzero elements in the $i$ th column of $\boldsymbol{P}$. Then, the geometrical selection criterion considering error propagation becomes

$$
\omega_{g-p}^{*}=\arg \min _{\omega \in \Omega}\left\{\max _{i \leq M_{T}} k_{i} \cdot Q\left(\frac{\sqrt{2}\left\|\boldsymbol{d}_{\omega, i}\right\|}{\sigma_{n}}\right)\right\} .
$$

\section{F. Simulation Results}

To show the improvement of employing antenna selection in LRA receivers, we consider a system with $M_{R}=M_{T}=2$ RF chains. We use 16 QAM symbols generated as $\Re\left\{s_{i}\right\} \in$ $\{-3,-1,1,3\}$ and $\Im\left\{s_{i}\right\} \in\{-1,-3,1,3\}$, i.e., $E\left\{s_{i} s_{i}^{*}\right\}=10$ $\mathrm{dB}$. We assume that the uncorrelated MIMO channel is randomly generated with $h_{i j} \sim \mathcal{N}_{c}(0,1)$, perfectly known at the receiver and remains constant for several data bursts. For comparison, the performance of the ML and the traditional ZF linear receiver without antenna selection is also considered. In Fig. 8, it is seen that without antenna selection, the ZF LRA receiver behaves better than the traditional ZF linear receiver and achieves a diversity similar to the one of the ML decoder. In the same figure, we consider antenna selection with ZF LRA receivers. We show the performance of the LRA receiver with $K_{R}=3$, $K_{R}=4, K_{R}=6$, and $K_{R}=8$ antennas. The optimal antenna subset is found by exhaustive search over all possible antenna subsets for which we have run long simulations to find the SER associated to each antenna subset in $\Omega$. It is seen in Fig. 8 that there is an improvement of several orders of magnitude by employing antenna selection at the receiver with a minimum additional complexity, cost, and hardware requirements. It is also observed that antenna selection increases both the diversity (i.e., the slope of the SER curve with respect to the SNR) and the coding gain with respect to the system without selection. Note that by employing only an additional antenna, i.e., $K_{R}=3$, the simple ZF LRA receiver already provides better performance than the optimal ML receiver with no antenna selection that, for 


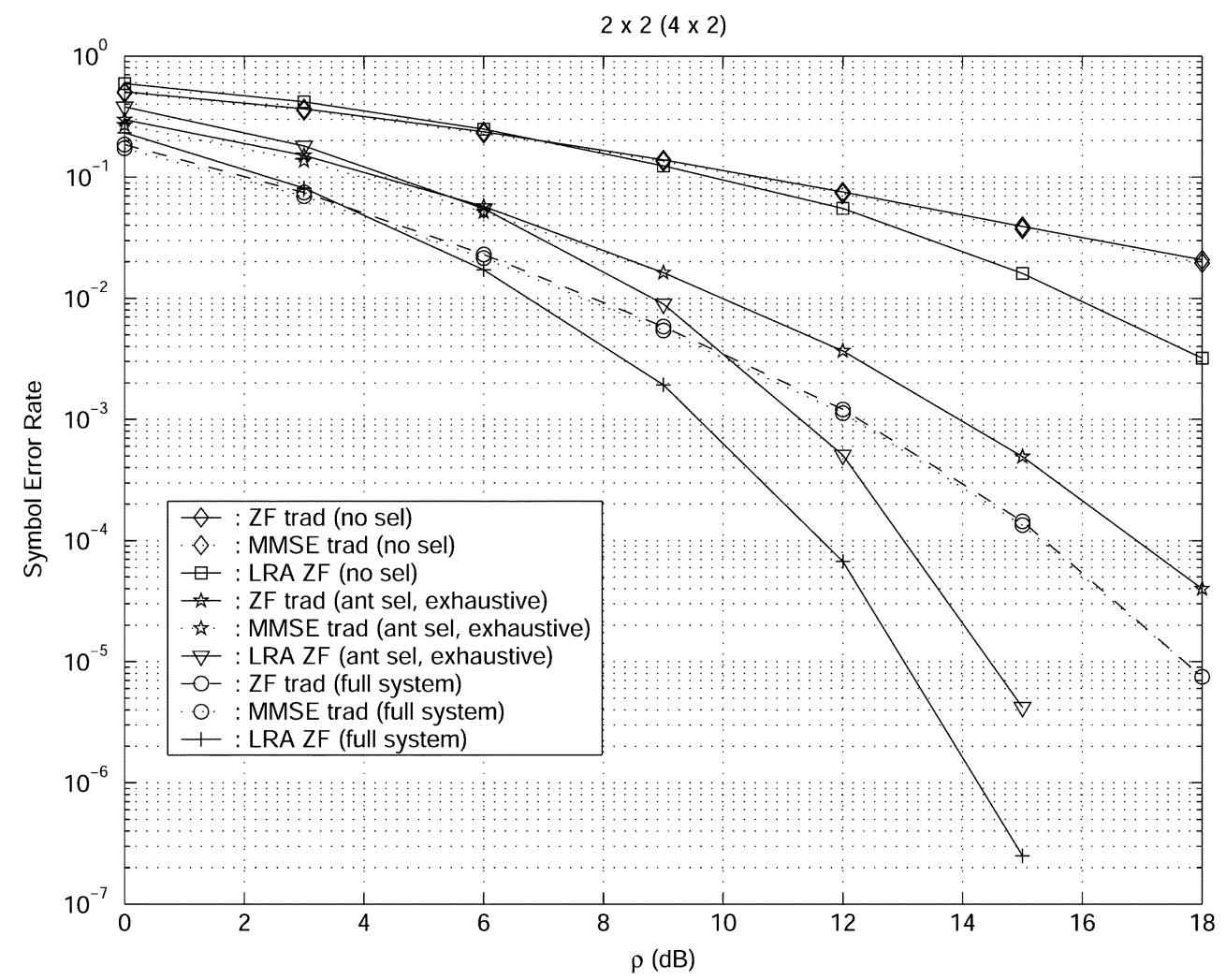

Fig. 9. Symbol ER comparison between full systems $(4 \times 2)$, antenna selection $(2 \times 2$ with four receive antennas to select $)$, and limited systems $(2 \times 2)$.

the 16-QAM constellation case, requires as much as $256 \mathrm{com}$ parisons for every decoded symbol. Therefore, a simple LRA linear receiver with antenna selection with only one extra antenna are good candidates to improve the MIMO performance while reducing the receiver complexity.

Fig. 9 compares the performance of antenna selection on traditional linear receivers (ZF and mmse) and LRA receivers when $M_{T}=2, M_{R}=2$, and $K_{R}=4$. The rest of the parameters are the same as before. We also consider the full system on both linear receivers and LRA receivers, i.e., when all the $K_{R}$ receive antennas are used. With two transmit antennas, ZF and mmse traditional linear receivers give similar performance and the curves almost overlap. It is seen that LRA ZF receivers with antenna selection can obtain better performance than the full system used with traditional linear receivers. Similar to space-time coding systems with antenna selection [8], the diversity of spatial multiplexing systems with antenna selection is the same as the diversity of the full system (the curves have the same slope). This result highly motivates the use of antenna selection in spatial multiplexing systems. However, there is a small loss in the coding gain. As in space-time coding systems [8], it has been observed that the loss is bounded by $10 \log \left(K_{R} / M_{R}\right)$.

We now compare the performance among the different antenna-selection criteria proposed in this paper. We first consider a system with $M_{T}=M_{R}=2$ and $K_{R}=6$ antennas. The SER associated with each antenna subset is found running long simulations and averaged among multiple channel realizations. It is seen in Fig. 10 that all the different criteria bound the SER very tightly. The mse and geometrical criteria considering error propagations are the best criteria for this task and have the same performance. The worst antenna-selection criteria in terms of SER correspond to the maximum mutual information and maximum minimum eigenmode. However, these criteria have the smallest computational complexity since they do not require a lattice basis reduction. Note that $\omega_{g}^{*}$ and $\omega_{\mathrm{mse}}^{*}$ criteria defined in (24) and (29), respectively, do not depend on the SNR of the channel and select the best antenna subset in most cases, which justifies our claim in Section IV.3 that, in general, the antenna subset minimizing the SER at high SNR is the same as the antenna subset minimizing the SER at low SNR.

We now consider a larger number of antennas, i.e., $M_{T}=$ $M_{R}=9$ and $K_{R}=12$. The reduction parameter in Algorithm 1 is $\delta=0.8$. In this scenario, there are 220 possible antenna subsets. Note that this is not the required number of different channel estimations, since by combining the rows of two different channel estimates we can have access to the 220 different channel matrices. The results are shown in Fig. 11. As expected, the performance of the mse and the optimal decision region criteria is the same (the curves completely overlap) with and without considering the error propagation. It is seen that the selection criteria that consider error propagation are the best selection criteria in terms of SER. When considering simple selection criteria, the mutual information criterion does not give a very good performance, whereas the minimum eigenmode gives slightly better results. Notice that with a ML decoding algorithm with 16-QAM symbols and nine transmit antennas, the receiver would require as much as $16^{9}>6 \cdot 10^{10}$ comparisons.

\section{AdAPtive Antenna Selection}

So far, we have assumed that the channel $\boldsymbol{H}_{\omega}$ is perfectly known at the receiver and based on it; different selection criteria 


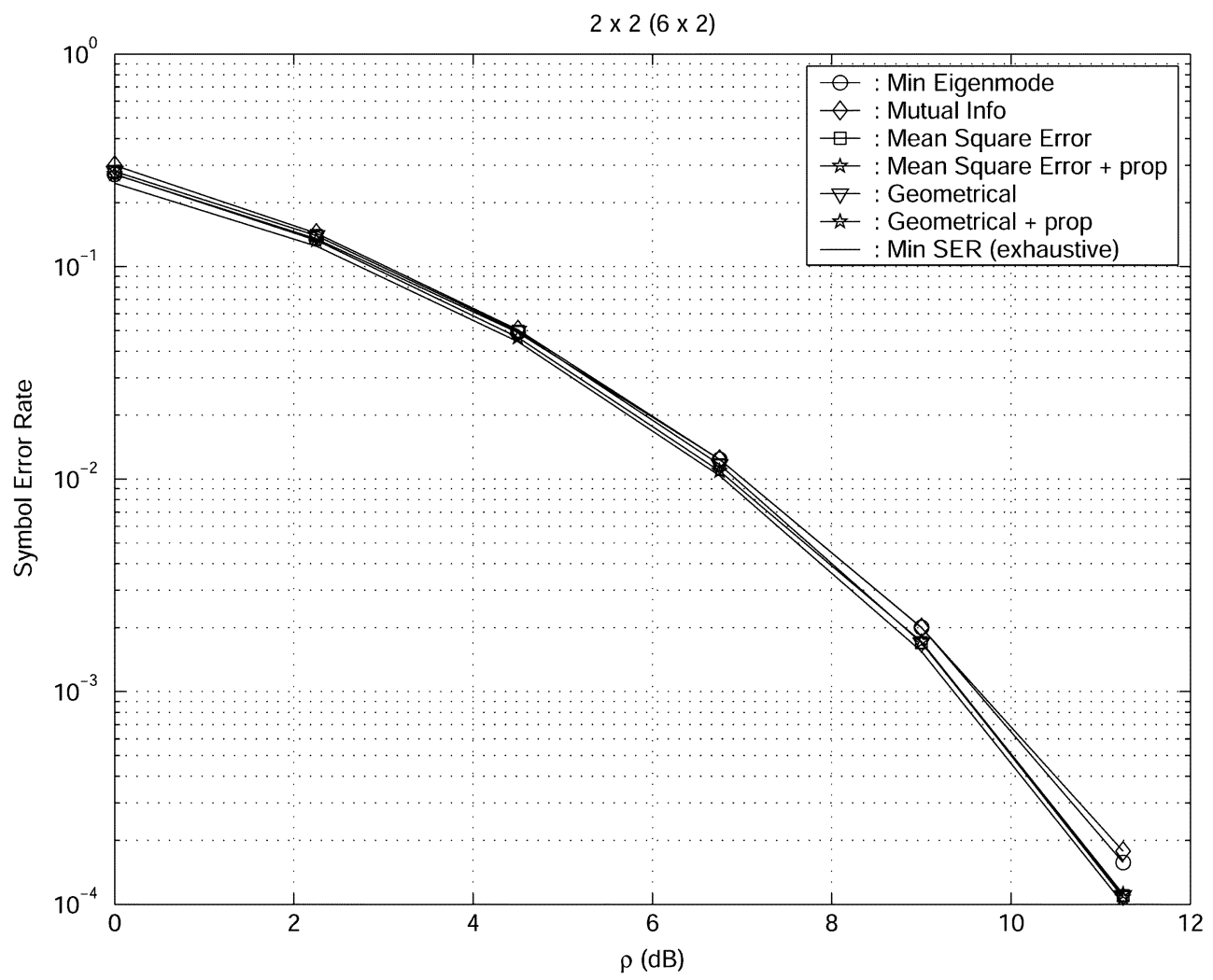

Fig. 10. Symbol ER of ZF LRA receivers employing different antenna-selection criteria in a $2 \times 2(6 \times 2)$ system.

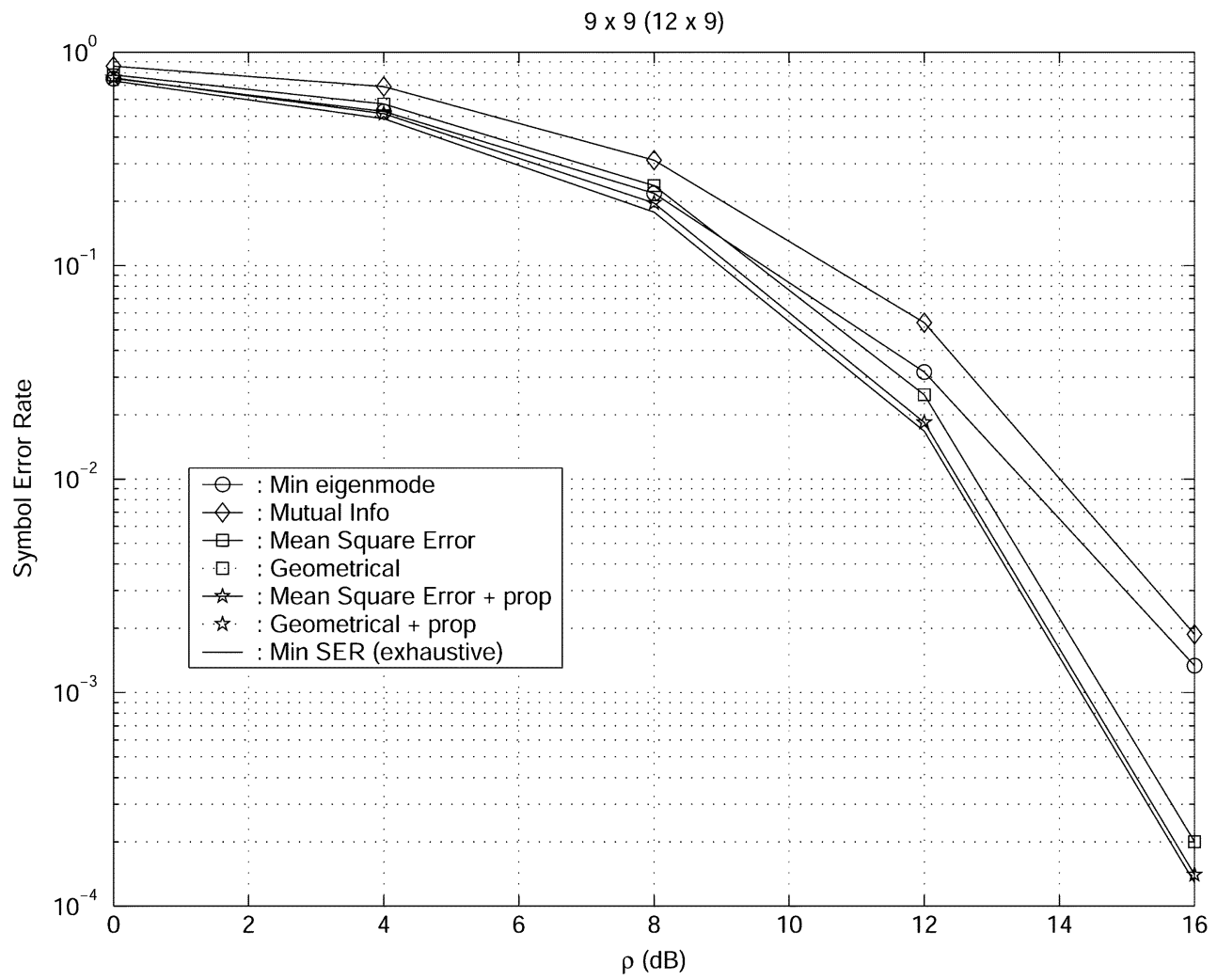

Fig. 11. Symbol ER of ZF LRA receivers employing different antenna-selection criteria in a $9 \times 9(12 \times 9)$ system.

perform an exhaustive search of an objective function evaluated over all possible antenna subsets. We now consider situations in which the receiver only has access to noisy estimates of the channel $\hat{\boldsymbol{H}}_{\omega}$ obtained using training sequences. Then, the objec- tive function in the different selection criteria cannot be evaluated analytically, but can only be estimated. In this case, we consider antenna selection using discrete stochastic approximation algorithms based on the discrete stochastic optimization tech- 
niques found in the recent operations research literature [19]. These techniques have recently been applied to solve several other problems in wireless communications [32]. These algorithms optimize an objective function $\Phi\left(\boldsymbol{H}_{\omega}\right)$ (e.g., maximum mutual information in Section IV.A, mmse in Section IV.D, or minimum error distance in Section IV.E) over a set of feasible solutions (e.g., antenna subsets to be used) when the objective function cannot be evaluated analytically, but can only be estimated. The algorithms take ideas from the traditional adaptive filtering algorithms such as the least-mean-squares (LMS) algorithm, in which at each iteration, the algorithm makes a move toward a better solution. But, in this case, the parameters to be optimized take discrete values (i.e., antenna indices to be used). The algorithm is useful in very slow-varying channels. In the antenna-selection application, it is applicable to fixed wireless access systems, for example.

We first consider channel estimation at the receiver using training sequences [33]. Suppose that $T \geq M_{T}$ MIMO training symbols are used to probe the channel. Then, the received signal corresponding to the training sequence can be written as

$$
\boldsymbol{Y}=\sqrt{\frac{\rho}{M_{T}}} \boldsymbol{H}_{\omega} \boldsymbol{S}+\boldsymbol{N}
$$

where $\boldsymbol{Y}$ is the $\left(M_{R} \times T\right)$ received matrix, $S$ is the $\left(M_{T} \times T\right)$ training sequence, and $N$ is the $\left(M_{R} \times T\right)$ received noise matrix. Then, the ML estimate of the channel matrix associated to the antennas subset $\omega, \hat{\boldsymbol{H}}_{\omega}$ is given by

$$
\begin{aligned}
\hat{\boldsymbol{H}}_{\omega} & =\arg \min _{\boldsymbol{H} \in \mathbb{C}^{M_{R} \times M_{T}}}\left\|\boldsymbol{Y}-\sqrt{\frac{\rho}{M_{T}}} \boldsymbol{H} \boldsymbol{S}\right\|^{2} \\
& =\sqrt{\frac{M_{T}}{\rho}} \boldsymbol{Y} \boldsymbol{S}^{H}\left(\boldsymbol{S} \boldsymbol{S}^{H}\right)^{-1} .
\end{aligned}
$$

According to [33], the optimal training symbol matrix $S$ to minimize the channel-estimation error should be orthogonal, i.e., $\boldsymbol{S} \boldsymbol{S}^{H}=T \boldsymbol{I}_{M_{T}}$.

Suppose that at time $n$ the receiver obtains an estimate of the channel $\hat{\boldsymbol{H}}_{\omega}[n]$ and computes a noisy estimate of the objective function $\Phi\left(\boldsymbol{H}_{\omega}\right)$ denoted as $\phi[n, \omega]$. Given a sequence of i.i.d. random variables $\{\phi[n, \omega], n=1,2, \ldots\}$, if each $\phi[n, \omega]$ is an unbiased estimate of the objective function $\Phi\left(\boldsymbol{H}_{\omega}\right)$, then the optimization problem can be reformulated as the following discrete stochastic optimization problem:

$$
\omega^{*}=\arg \max _{\omega \in \Omega} \Phi\left(\boldsymbol{H}_{\omega}\right)=\arg \max _{\omega \in \Omega} E\{\phi[n, \omega]\} .
$$

To solve the problem in (34), we now present a globally convergent stochastic approximation algorithm based on [19]. We use the $P=\left(\begin{array}{l}K_{R} \\ M_{R}\end{array}\right)$ unit vectors as labels for the $P$ possible antenna subsets, i.e., $\Xi=\left\{\boldsymbol{e}_{1}, \boldsymbol{e}_{2}, \ldots, \boldsymbol{e}_{P}\right\}$, where $\boldsymbol{e}_{i}$ denotes the $(P \times 1)$ vector with a one in the $i$ th position and zeros elsewhere. At each iteration, the algorithm updates the $(P \times 1)$ probability vector $\pi[n]=[\pi[n, 1], \ldots, \pi[n, P]]^{T}$ representing the state occupation probabilities with elements $\pi[n, i] \in[0,1]$ and $\sum_{i} \pi[n, i]=1$. Let $\omega^{(n)}$ be the antenna subset chosen at the $n$th iteration. For notational simplicity, it is convenient to map the sequence of antenna subsets $\left\{\omega^{(n)}\right\}$ to the sequence $\{\boldsymbol{D}[n]\} \in \Xi$ of unit vectors where $\boldsymbol{D}[n]=\boldsymbol{e}_{i}$ if $\omega^{(n)}=\omega_{i}$, $i=1, \ldots, P$.

Algorithm 2 Discrete stochastic approximation algorithm for antenna selection

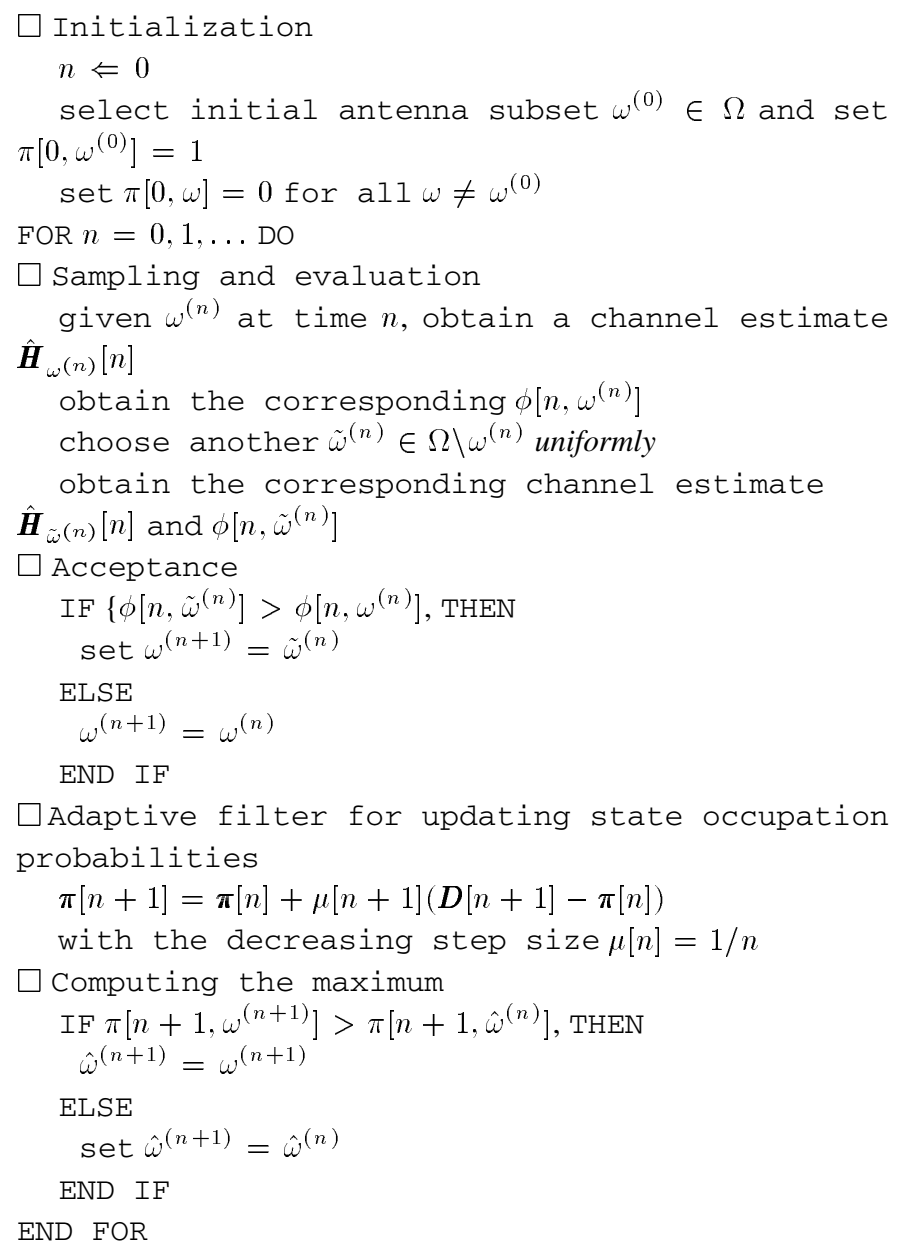

Under certain conditions [19], the sequence $\left\{\hat{\omega}^{(n)}\right\}$ almost surely converges to the global maximizer $\omega^{*}$. Therefore, $\hat{\omega}^{(n)}$ denotes the estimate at time $n$ of the optimal antenna subset $\omega^{*}$. If we denote $W^{(n)}[\omega]$ for each $\omega \in \Omega$ as a counter of the number of times the Markov chain has visited antenna subset $\omega^{(n)}$ by time $n$, we can observe that $\pi[n]=(1 / n)\left[W^{(n)}\left[\omega_{1}\right], \ldots, W^{(n)}\left[\omega_{P}\right]\right]^{T}$. Therefore, the algorithm chooses the antenna subset that has been visited most often by the Markov chain $\left\{\omega^{(n)}\right\}$ so far.

\section{A. Simulation Results}

To illustrate the convergence of Algorithm 2 in static channels, we consider the antenna-selection criterion based on the optimal geometrical decision region, which gives a good bound on the ER, as shown in Section V. Define $\left\|\boldsymbol{d}_{\omega}\right\|=\min _{1 \leq i \leq M_{T}}\left\|\boldsymbol{d}_{\omega, i}\right\|$. Then, the objective function to be maximized becomes $\Phi\left(\boldsymbol{H}_{\omega}\right)=\left\|\boldsymbol{d}_{\omega}\right\|^{2}$. Suppose that at time $n$ the receiver obtains an estimate of the channel $\hat{\boldsymbol{H}}_{\omega}[n]$, when the antenna subset chosen is $\omega$. Then, the estimate of the lattice 
$\|\mathrm{d}\|$ of chosen antenna set vs iteration number

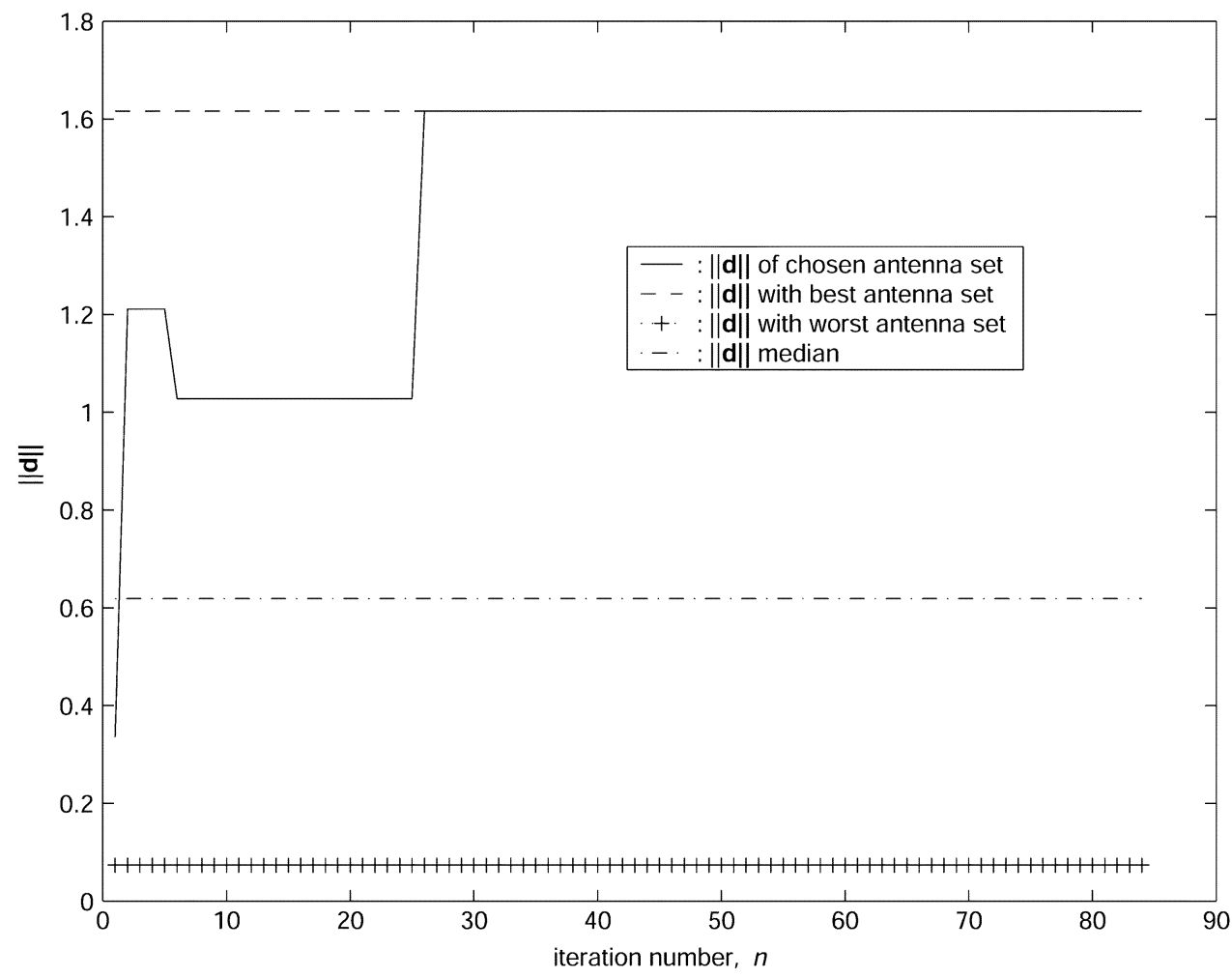

Fig. 12. Single run of the algorithm: $\left\|\boldsymbol{d}_{\omega}\right\|$ of the chosen antenna subset versus time $n$.

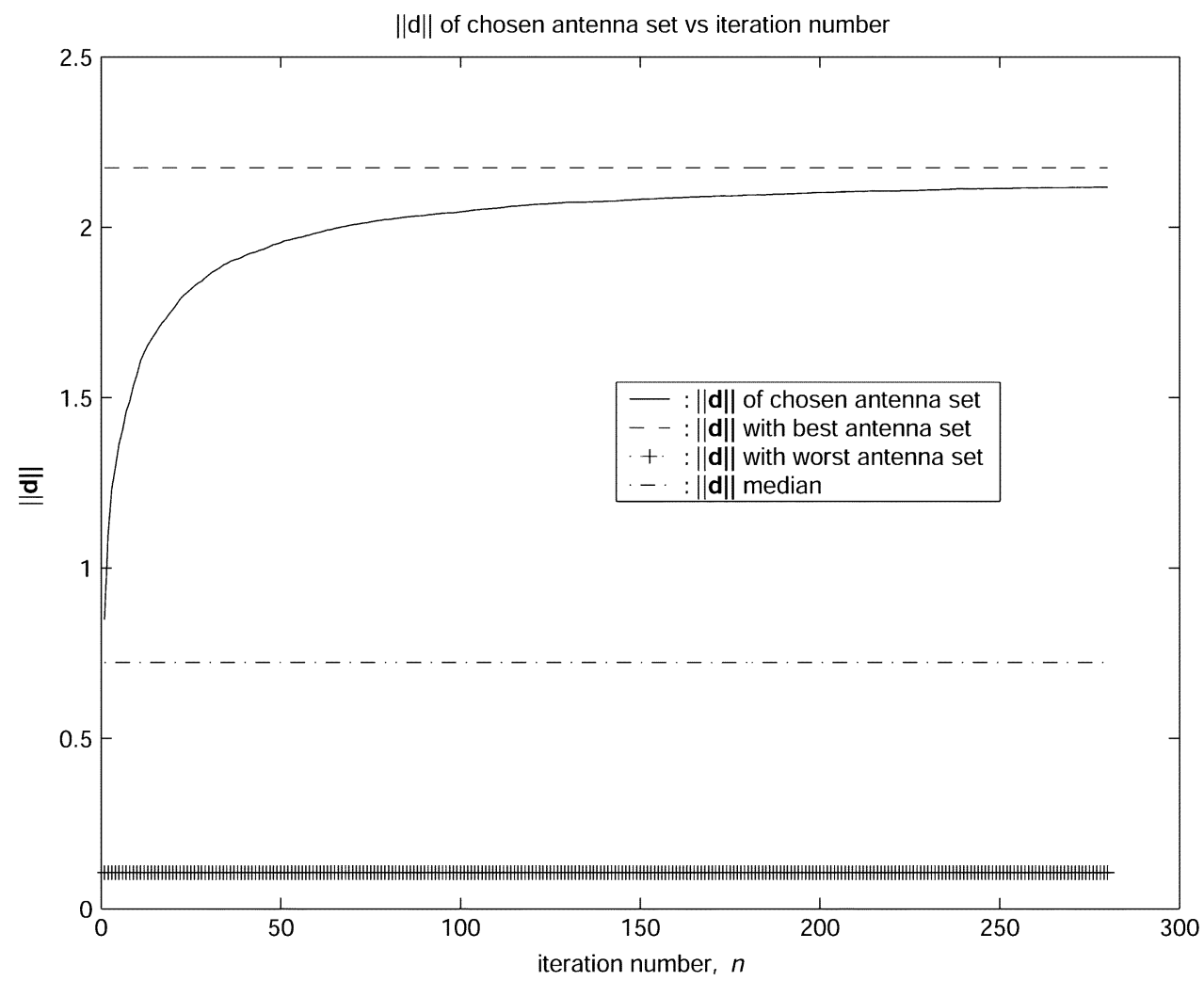

Fig. 13. Average of 280 iterations of the algorithm over 100 different MIMO channel realizations: $\left\|\boldsymbol{d}_{\omega}\right\|^{2}$ of the chosen antenna subset versus time $n$.

reduced basis becomes $\hat{\boldsymbol{H}}_{\omega}^{\prime}[n]$ and Algorithm 2 uses a noisy estimate of the objective function $\left\|\boldsymbol{d}_{\omega}\right\|$ as

$$
\phi[n, \omega]=\min _{1 \leq i \leq M_{T}} \frac{1}{2}\left\|\pi_{i}\left(\hat{\boldsymbol{h}}_{\omega, i}^{\prime}[n]\right)\right\|
$$

where $\hat{\boldsymbol{h}}_{\omega, i}^{\prime}[n]$ denotes the $i$ th column of $\hat{\boldsymbol{H}}_{\omega}^{\prime}[n]$. For the simulations, we consider $M_{R}=M_{T}=2$ and $K_{R}=8$ antennas. We use the ML channel estimate in (33) with $T=4$ orthogonal training symbols. We set $\rho=0 \mathrm{~dB}$ and $E\left\{s_{i}^{*} s_{i}\right\}=10 \mathrm{~dB}$. The 
$\left(K_{R} \times M_{T}\right)$ channel $\boldsymbol{H}$ is randomly generated and fixed during the whole simulation. Fig. 12 shows a single run of the algorithm with 84 iterations. In this same figure, we show the distance $\left\|\boldsymbol{d}_{\omega}\right\|$ of the best antenna subset and the worst antenna subset, as well as the median distance among the $\left(\begin{array}{l}8 \\ 2\end{array}\right)=28$ antenna configurations, found by exhaustive search. Next, in Fig. 13 we consider 280 iterations in each execution and average the distance $\left\|\boldsymbol{d}_{\omega}\right\|$ of the antenna subset selected at each iteration by the algorithm over 100 different channel realizations. From both figures, it is seen that the algorithm adaptively moves to the best antenna subset. We observe that although the algorithm takes some time to converge to the optimal antenna subset, it very quickly moves to an antenna subset with a large value of $\left\|\boldsymbol{d}_{\omega}\right\|$.

\section{CONCLUSION}

In this paper, we have proposed different antenna-selection criteria for lattice-aided-reduction linear receivers. The insights of these kind of receivers have been described to understand how the selection criteria are related to the operations at the receiver. The performance of the different antenna-selection criteria has been compared in terms of symbol ER and it has been observed that we can obtain an improvement of several orders of magnitude by considering antenna selection at the receiver that does not require any increase of decoding complexity and only very little extra hardware. Moreover, we have developed adaptive versions of the antenna-selection algorithms in situations where only noisy estimates of the channel are available. Simulation results have demonstrated excellent convergence properties of these algorithms.

\section{REFERENCES}

[1] G. J. Foschini and M. J. Gans, "On the limits of wireless communications in a fading environment when using multiple antennas," Wireless Pers. Commun., vol. 6, pp. 311-335, 1998.

[2] I. E. Telatar, "Capacity of multi-antenna Gaussian channels," Eur. Trans. Telecommun., vol. 10, pp. 585-595, 1999.

[3] R. Nabar, D. Gore, and A. Paulraj, "Optimal selection and use of transmit antennas in wireless systems," presented at the Int. Conf. Telecommun. (ICT'00), Acapulco, Mexico, May 2000.

[4] A. Gorokhov, "Antenna selection algorithms for MEA transmission systems," in Proc. IEEE Int. Conf. Acoustics, Speech, Signal Processing (ICASSP'02), Orlando, FL, May 2002, pp. 2857-2860.

[5] A. Gorokhov, D. A. Gore, and A. J. Paulraj, "Receive antenna selection for MIMO flat-fading channels: Theory and algorithms," IEEE Trans. Inform. Theory, vol. 49, pp. 2867-2696, Oct. 2003.

[6] D. A. Gore and A. J. Paulraj, "MIMO antenna subset selection with space-time coding," IEEE Trans. Signal Processing, vol. 50, pp. 2580-2588, Oct. 2002.

[7] D. Gore and A. Paulraj, "Statistical MIMO antenna sub-set selection with space-time coding," in Proc. IEEE Int. Conf. Communications (ICC'02), New York, Apr. 2002, pp. 641-645.

[8] A. Ghrayeb and T. M. Duman, "Performance analysis of MIMO systems with antenna selection over quasistatic fading channels," IEEE Trans. Veh. Technol., vol. 58, pp. 281-288, Mar. 2003.

[9] I. Bahceci, T. M. Duman, and Y. Altunbasak, "Antenna selection for multiple-antenna transmission systems: Performance analysis and code construction," IEEE Trans. Inform. Theory, vol. 49, pp. 2669-2681, Oct. 2003.

[10] W. H. Wong and E. G. Larsson, "Orthogonal space-time block coding with antenna selection and power allocation," Electron. Lett., vol. 39, pp. 379-381, 2003.

[11] R. W. Heath, Jr., S. Sandhu, and A. Paulraj, "Antenna selection for spatial multiplexing systems with linear receivers," IEEE Commun. Lett., vol. 5, pp. 142-144, Apr. 2001.
[12] A. Gorokhov, D. A. Gore, and A. J. Paulraj, "Receive antenna selection for MIMO spatial multiplexing: Theory and algorithms," IEEE Trans. Signal Processing, vol. 51, pp. 2796-2807, Nov. 2003.

[13] R. S. Blum and J. H. Winters, "On optimum MIMO with antenna selection," IEEE Commun. Lett., vol. 6, pp. 322-324, Aug. 2002.

[14] Z. Chen, B. Vucetic, and J. Yuan, "Space-time trellis codes with transmit antenna selection," Electron. Lett., vol. 39, pp. 854-855, 2003.

[15] A. Molisch, M. Win, and J. Winters, "Capacity of MIMO systems with antenna selection," in Proc. IEEE Int. Conf. Commun. (ICC'01), Helsinki, Finland, June 2001, pp. 570-574.

[16] A. F. Molisch, "MIMO systems with antenna selection-An overview," in Proc. IEEE Radio Wireless Conf. (RAWCON'03), Boston, MA, Aug. 2003, pp. 167-170.

[17] P. J. Voltz, "Characterization of the optimum transmitter correlation matrix for MIMO with antenna subset selection," IEEE Trans. Commun., vol. 51, pp. 1779-1782, Nov. 2003.

[18] H. Yao and G. W. Wornell, "Lattice-reduction-aided detectors for MIMO communication systems," in Proc. IEEE GLOBECOM'02, Taipei, Taiwan, Nov. 2002, pp. 424-428.

[19] S. Andradottir, "A global search method for discrete stochastic optimization," SIAM J. Optim., vol. 6, pp. 513-530, 1996.

[20] G. D. Golden, G. J. Foschini, R. A. Valenzuela, and P. W. Wolniansky, "Detection algorithm and initial laboratory results using the V-BLAST space-time communication architecture," Electron. Lett., vol. 35, pp. 14-15, 1999.

[21] L. Lovasz, An Algorithmic Theory of Numbers, Graphs, and Convexity. Philadelphia, PA: SIAM, 1986.

[22] C. P. Schnorr, "A more efficient algorithm for lattice basis reduction," $J$. Algorithms, vol. 9, pp. 47-62, 1988.

[23] C. P. Schnorr and M. Euchner, "Lattice basis reduction: Improved practical algorithms and solving subset sum problems," Math. Program., vol. 66, pp. 181-191, 1994.

[24] A. H. Banihashemi and A. K. Khandani, "On the complexity of decoding lattices using the Korkin-Zolotarev reduced basis," IEEE Trans. Inform. Theory, vol. 44, pp. 162-171, Jan. 1998.

[25] A. Korkin and G. Zolotarev, "Sur les formes quadratiques," Math. Ann., vol. 6, pp. 366-389, 1873.

[26] J. C. Lagarias, H. W. Lenstra, and C. P. Schnorr, "Korkin-Zolotarev bases and successive minima of a lattice and its reciprocal," Combinatorica, vol. 10, pp. 333-348, 1990.

[27] L. Afflerback and H. Grothe, "Calculation of Minkowski-reduced lattice bases," Comput., vol. 35, pp. 269-276, 1985.

[28] B. Helfrich, "Algorithms to construct Minkowski reduced and Hermite reduced lattice bases," Theor. Comput. Sci., vol. 41, pp. 125-139, 1985.

[29] B. A. LaMacchia, "Basis reduction algorithms and subset sum problems," SM thesis, Massachusetts Inst. Technol., Cambridge, 1991.

[30] M. Seysen, "Simultaneous reduction of a lattice basis and its reciprocal basis," Combinatorica, vol. 13, pp. 363-376, 1993.

[31] C. P. Schnorr, "A hierarchy of polynomial time lattice basis reduction algorithms," Theor. Comput. Sci., vol. 53, pp. 201-224, 1987.

[32] V. Krishnamurthy, X. Wang, and G. Yin, "Spreading code optimization and adaptation in CDMA via discrete stochastic approximation," IEEE Trans. Inform. Theory, to be published.

[33] T. L. Marzetta, "BLAST training: Estimation channel characteristics for high-capacity space-time wireless," in Proc. 37th Annu. Allerton Conf. Communications, Computing, Control, Monticello, IL, Sept. 1999, pp. 958-966.

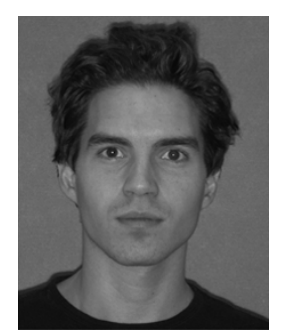

Inaki Berenguer received the Telecommunications Engineer degree from the Technical University of Valencia (UPV), Valencia, Spain, in 2000 and the M.Phil. degree in electrical engineering from Cambridge University, Cambridge, U.K., in 2002. $\mathrm{He}$ is currently working toward the Ph.D. degree in electrical engineering at the University of Cambridge, U.K., and Columbia University, New York, as a two-year Fulbright scholar.

From July 1999 to January 2001, he was with Hewlett Packard, Brussels, Belgium. In January 2001, he joined the Wireless Division, STMicroelectronics, San Diego, CA, where he worked on a W-CDMA project until August 2001. His research interests include communications theory and digital signal processing.

Mr. Berenguer also is a Recipient of the British Council-La Caixa, Cambridge-MIT Institute, and Leonardo Da Vinci fellowships. 


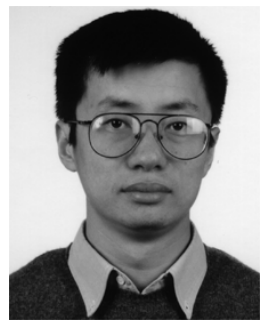

Xiaodong Wang (S'98-M'98) received the B.S. degree in electrical engineering and applied mathematics (with the highest honor) from Shanghai Jiao Tong University, Shanghai, China, in 1992, the M.S. degree in electrical and computer engineering from Purdue University, West Lafayette, IN, in 1995, and the Ph.D. degree in electrical engineering from Princeton University, Princeton, NJ, in 1998.

From July 1998 to December 2001, he was an Assistant Professor in the Department of Electrical Engineering, Texas A\&M University, College Station. In January 2002, he joined the Faculty of the Department of Electrical Engineering, Columbia University, New York. His research interests fall in the general areas of computing, signal processing, and communications. He has worked in the areas of digital communications, digital signal processing, parallel and distributed computing, nanoelectronics, and bioinformatics; he has published extensively in these areas. Among his publications is a recent book entitled Wireless Communication Systems: Advanced Techniques for Signal Reception (Englewood Cliffs, NJ: Prentice-Hall, 2003). His current research interests include wireless communications, Monte Carlo-based statistical signal processing, and genomic signal processing.

Dr. Wang received the 1999 National Science Foundation CAREER Award and the 2001 IEEE Communications Society and Information Theory Society Joint Paper Award. He currently serves as an Associate Editor for the IEEE TRANSACTIONS ON COMMUNICATIONS, the IEEE TRANSACTIONS ON WIRELESS Communications, the IEEE Transactions on Signal Processing, and the IEEE TRANSACTIONS ON INFORMATION THEORY. 